\title{
Striated Populations in Disordered Environments with Advection
}

Thiparat Chotibut ${ }^{\text {田 }}$ and David R. Nelson ${ }^{\text {甲 }}$

Department of Physics, Harvard University, Cambridge, Massachusetts 02138, USA

\section{Sauro Succi ${ }^{\boxplus}$}

Istituto per le Applicazioni del Calcolo,

Rome Via dei Taurini 19, 00185, Roma, Italy, and

Institute for Applied Computational Science,

John A. Paulson School of Engineering and Applied Science,

Harvard University, Cambridge, Massachusetts 02138, USA

(Dated: June 24, 2016) 


\begin{abstract}
Growth in static and controlled environments such as a Petri dish can be used to study the spatial population dynamics of microorganisms. However, natural populations such as marine microbes experience fluid advection and often grow up in heterogeneous environments. We investigate a generalized Fisher-Kolmogorov-Petrovsky-Piscounov (FKPP) equation describing single species population subject to a constant flow field and quenched random spatially inhomogeneous growth rates with a fertile overall growth condition. We analytically and numerically demonstrate that the non-equilibrium steady-state population density develops a flow-driven striation pattern. The striations are highly asymmetric with a longitudinal correlation length that diverges linearly with the flow speed and a transverse correlation length that approaches a finite velocity-independent value. Linear response theory is developed to study the statistics of the steady states. Theoretical predictions show excellent agreement with the numerical steady states of the generalized FKPP equation obtained from Lattice Boltzmann simulations. These findings suggest that, although the growth disorder can be spatially uncorrelated, correlated population structures with striations emerge naturally at sufficiently strong advection.
\end{abstract}

PACS numbers: 87.23.Cc, 87.18.Tt, 82.40.Ck, 05.40.-a

Keywords: population dynamics, spatially quenched disorder, generalized FKPP equation, pattern formation

\footnotetext{
* Electronic address: thiparatc(agmail.com

$\dagger$ Electronic address: nelson $@$ physics.harvard.edu

$\ddagger$ Electronic address: succi@iac.cnr.it
} 


\section{CONTENTS}

L. Introduction 3

ㄴ. Steady-state density from the linear response approximation 8

A. Density modulation due to a point-like growth hot spot 10

ㅍ. Longitudinal striations in the steady state for strong advection in $d \geq 2 \quad 14$

A. Two-point correlation function and the striation pattern 14

B. Comparisons to numerical simulations 18

IV. Conclusions and outlook 21

Acknowledgments 24

A. Green's Functions, correlation functions, and structure factors 24

$\begin{array}{lr}\text { References } & 27\end{array}$

\section{INTRODUCTION}

How growth, competition, and dispersal affect the spatial structure of living populations has been a keystone to understanding biodiversity and stability of ecosystems [1-5]. Numerous works demonstrate that simple dispersal mechanisms, such as diffusive spreading and chemotaxis, when coupled with spatial heterogeneity of resources, may result in intricate farfrom-equilibrium spatial patterning in diverse populations, including, for example, bacterial patterns on a Petri dish [6-8], soft-sediment mussel beds [9-11], and vegetation patterns in arid ecosystems [4, 12, 13]. More complicated dispersal mechanisms such as advective transport may also influence spatial distributions in natural populations. For instance, patchiness and filamentation of planktonic communities on ocean surfaces can arise from the delicate interplay between growth, competition, diffusion, and advection by turbulence and chaotic oceanic flows [10, 14-16]. Two-dimensional compressible turbulence restricts growth and competition to thin filaments leading to a dramatic decrease in the global carrying capacity in model planktonic populations [17, 18]. Even in a simple effectively unidirectional flow field, the combined effect of spatial variations in the resource and advection on the spatial 
structure of populations is very rich [1.9-2.9].

A general framework for systematic exploration of the growth and diffusion of singlespecies populations in an advective, spatially-varying growth environments is the reactiondiffusion-advection equation [3, 30]:

$$
\partial_{t} c+\nabla \cdot[\mathbf{v}(\boldsymbol{x}, t) c]=\nabla \cdot[D(\boldsymbol{x}, t) \nabla c]+f[c] .
$$

In an ecological context, Eq. (U) prescribes the time evolution of the coarse-grained density $c \equiv c(\boldsymbol{x}, t)$ of populations consisting of individuals that are transported by advection with the velocity field $\mathbf{v}(\boldsymbol{x}, t)$, diffuses (for example by, say, a run and tumble mechanism in the case of bacteria) with a space and time-dependent diffusivity $D(\boldsymbol{x}, t)$, and reproduce with the density-dependent growth rate $f[c]$ that depends on a local environment. When advection is absent, the diffusivity is constant $D(\boldsymbol{x}, t)=D$, and the resources necessary for growth are homogeneously distributed, the density of populations that reproduce with the logistic growth rate $f[c]=a c-b c^{2}$ (with $a$ and $b$ constant) obey the well-known Fisher-KolmogorovPetrovsky-Piscounov (FKPP) equation:

$$
\partial_{t} c=D \nabla^{2} c+a c-b c^{2},
$$

which, for a uniform positive growth rate $a$, admits a stable non-linear wave solution describing a spatial range expansion of populations that advance from a region saturated at the local carrying capacity $K \equiv a / b$, into unoccupied territory [3, 31-33]. At long times, reproductive growth and diffusion fill up and saturate the domain; the population density becomes featureless and is equal to the carrying capacity $K$, which is the stable steady state of Eq. (2).

In contrast, interesting phenomena and non-trivial spatial population structure at long times arise when a constant flow field $\mathbf{v}(\boldsymbol{x}, t)=\mathbf{v}$ advects the population across a spatially quenched growth landscape of the form $f[c]=a(\boldsymbol{x}) c-b c^{2}$ [1.9-21, 23]. This minimal extension of the FKPP equation reads

$$
\partial_{t} c+\mathbf{v} \cdot \nabla c=D \nabla^{2} c+a(\boldsymbol{x}) c-b c^{2} .
$$

Since advection can wash away the populations from the favourable growth hot spots and 
diffusion tends to spread populations out, the density $c(\boldsymbol{x}, t)$ deviates from the profile of the local carrying capacity $K(\boldsymbol{x}) \equiv a(\boldsymbol{x}) / b$ in regions where $a(\boldsymbol{x})>0$. For instance, in a landscape with a finite-size favourable growth hot spot surrounded by an unfavourable growth environment, the long-time population structure that would otherwise localize near the hot spot, can be driven by advection to delocalize or becomes extinct [21]. This localizationextinction transition has been observed in microbial experiments [24, 34]. In addition, in a random environment where the local growth rate can be regarded as a spatially quenched time-independent random variable, early-time growth eigenfunctions of the linearized growth operator of Eq. (3) can exhibit a localization-delocalization transition, with a remarkable associated non-Hermitian spectral property [23, 28, 35-38]. In strongly advective environments in which the spatially averaged growth rate is positive, all the growth eigenfunctions are delocalized and the spreading dynamics of populations at the frontier in the direction transverse to the flow is expected to be super-diffusive [22]].

While the early-time properties of growth eigenfunctions and growth dynamics in a spatially quenched random growth rate are relatively well understood, less is known about the structure of long-time steady-state populations. Close to extinction ( in an overall hostile growth environment in which the growth rates $a(\boldsymbol{x})$ are mostly negative), when the earlytime growth eigenfunctions do not significantly overlap, the population structure at long times is tracked by the early-time growth eigenfunctions. However, more generally, significant changes due to the mode-coupling triggered by the non-linear saturation term $-b c^{2}$ distort this picture, especially when advection is strong [23].

In this work, with the goal of determining how constant advection combines with spatial variations in an overall fertile growth landscape to affect the long-time population structure, we analytically and numerically study steady-state populations described by Eq. (3). Specifically, we study the long-time steady-state population density $c^{*}(\boldsymbol{x}) \equiv c(\boldsymbol{x}, t \rightarrow \infty)$ satisfying the nonlinear equation,

$$
0=D \nabla^{2} c^{*}(\boldsymbol{x})-\mathbf{v} \cdot \nabla c^{*}(\boldsymbol{x})+a(\boldsymbol{x}) c^{*}(\boldsymbol{x})-b c^{* 2}(\boldsymbol{x}),
$$

arising from a weak spatially-quenched random growth landscape of the form

$$
a(\boldsymbol{x}) \equiv a_{0}+\delta a(\boldsymbol{x})
$$


where $a_{0}$ is a constant positive background growth rate, and $\delta a(\boldsymbol{x})$ is a weak frozen-in spatial perturbation such that $\langle a(\boldsymbol{x})\rangle>0$, where $\langle\cdot\rangle$ represents a spatial average. In the calculations that follow, we shall draw $\delta a(\boldsymbol{x})$ from a uniform, symmetrical box distribution, $\delta a(\boldsymbol{x}) \in[-\Delta, \Delta]$. Weak disorder then means $|\delta a(\boldsymbol{x})| / a_{0} \ll 1$. Although recent investigations suggest that demographic fluctuations due to stochasticity in the discrete birth and death events can be important close to extinction or in diluted populations [3.9-42], we focus here on the regime where the local carrying capacity $K(\boldsymbol{x})=a(\boldsymbol{x}) / b$ is large, so that demographic fluctuations are small and the mean field description of the steady state embodied in Eq.(四) is reliable.

In Sec. ㅁ. we show that the steady-state density fluctuations from the mean carrying capacity $\bar{K} \equiv a_{0} / b$ can be regarded as a linear response to a small perturbation caused by the quenched random background growth rate. The Green's function of the associated reactiondiffusion-advection operator, which we calculate in $d$ dimensions, describes the steady-state density response to a point-like growth hot spot. In the absence of advection, the response decays isotropically, with the diffusive localization length $\xi_{D} \equiv \sqrt{D / a_{0}}$, from the center of a growth hot spot. We then discuss how advection breaks isotropy: advection elongates (shortens) the downstream (upstream) longitudinal localization length, while symmetrically contracting the transverse localization length. Figs. 2 and 3 illustrate this effect, in $d=1$ and $d=2$, respectively. For strong advection such that $\tilde{\mathrm{v}} \equiv \mathrm{v} / \mathrm{v}_{F} \gg 1$ where $\mathrm{v}_{F} \equiv$ $2 \sqrt{D a_{0}}$ is the characteristic Fisher speed associated with the average growth rate [3, 33], the downstream and upstream longitudinal localization lengths scale as $\xi_{\|}^{+} \approx \tilde{\mathrm{v}} \xi_{D}=\mathrm{v} / 2 a_{0}$ and $\xi_{\|}^{-} \approx(1 / \tilde{\mathrm{v}}) \xi_{D}=2 D / \mathrm{v}$, while the transverse localization length scales as $\xi_{\perp} \approx(1 / \tilde{\mathrm{v}}) \xi_{D}$. The asymmetric elongation and contraction occur simultaneously with the decay in the response amplitude, a consequence of the conservation of density fluctuations discussed at the end of Sec. 미.

In Sec. $\mathbb{U}$, we show that advection and spatially quenched uncorrelated random growth rates together lead to striated patterns of steady-state population density in two dimensions, as depicted in Fig. [1. Although the growth rate fluctuations are spatially uncorrelated and the transverse localization length of the response from an isolated growth hot spot shrinks to zero as advection becomes stronger, a finite transverse correlation length of the steady state density nevertheless emerges at strong advection. These disorder-induced striated patterns arise from long-range advection, in contrast to the standard disorder-induced patterns in 
(a)

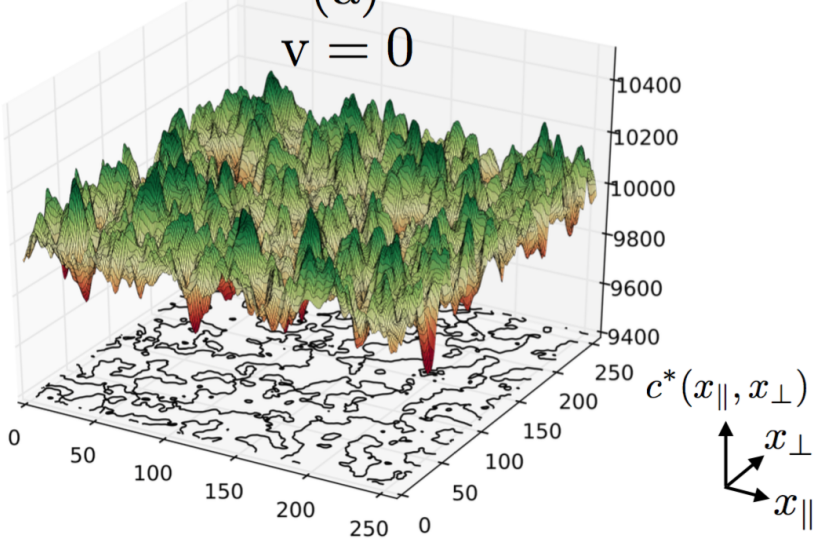

(b)

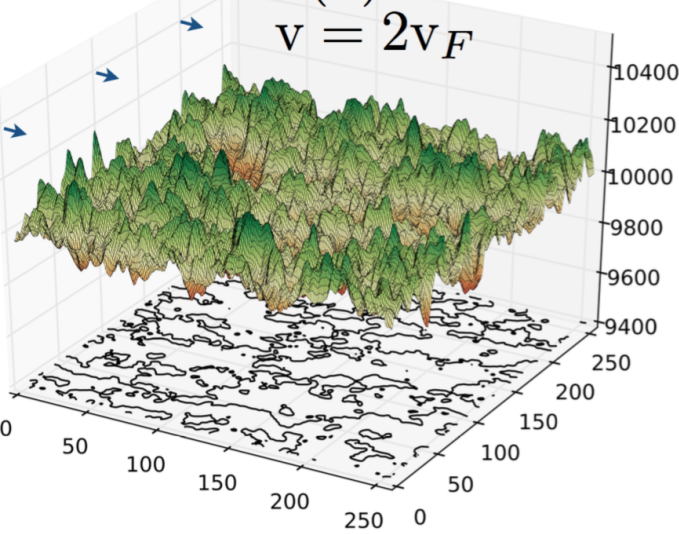

(d)

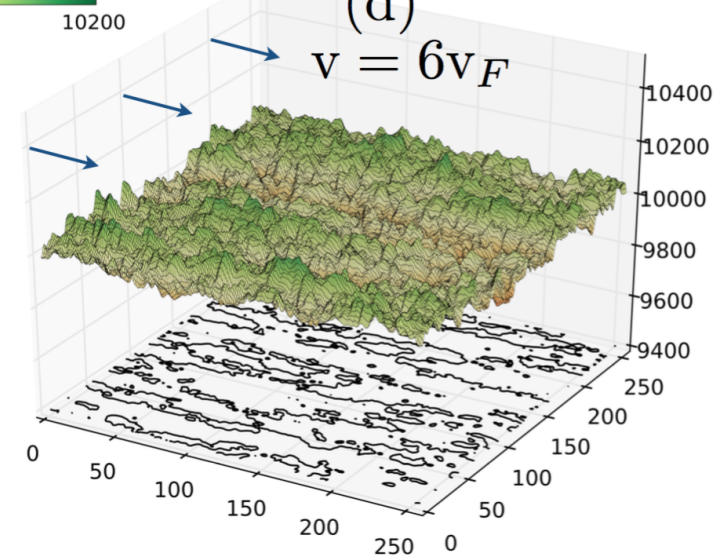

FIG. 1. (Color online) Typical long-time steady states $c^{*}(\boldsymbol{x})$ with striations in two dimensions that arise from the generalized FKPP equation with periodic boundary conditions and variable strength advection in the $+\hat{x}_{\|}$direction and with the same realization of a weak spatially quenched random growth rate. The black lines at the bottom indicate contours of $c^{*}(\boldsymbol{x})$ at the value of the mean carrying capacity $\bar{K}=10^{4}$, separating the more favourable growth domains from the less favourable ones. These plots result from the lattice Boltzmann simulation discussed in Sec. एWB. Without advection, as in (a), steady state density exhibit mild fluctuations around the mean carrying capacity $\bar{K}=10^{4}$. The correlations of the density fluctuations without advection are isotropic. However, advection breaks statistical isotropy of the steady state; correlation length is developed and is elongated in the longitudinal direction, as in (b), (c) and (d). Striated patterns emerge at strong flow $\mathrm{v} \gg \mathrm{v}_{F}$ with the longitudinal correlation length of order the system size while the transverse correlation length remains finite, see (d). Note also that strong advection lowers the amplitude of the density fluctuations.

systems with short-range transport, such as in the Swift-Hohenberg equation [43-45] or in Anderson localization problems [46]. In addition, striated steady-state patterns are static, unlike transient spatiotemporal patterns that appear in reaction-diffusion systems with demographic noises [47, 48]. The steady state disorder problem treated here is derived from the 
study of the FKPP equation with disordered growth rates in Ref. [23]. Theoretical analysis of the two-point correlation function leading to striated population structures is provided in Sec. WA. There, we also show that, for strong advection $\mathrm{v} \gg \mathrm{v}_{F}$, the longitudinal correlation length elongates without bound as $\xi_{\|}=\mathrm{v} / a_{0}$ whereas the transverse correlation length approaches the limiting value $\xi_{\perp}=\xi_{D}=\sqrt{D / a_{0}}$. Consequently, the population structure becomes highly anisotropic, with the ratio of correlation lengths given by $\xi_{\|} / \xi_{\perp}=2 \tilde{\mathrm{v}}$. Sec. [WB compares the long distance correlations from theoretical analysis with those from the lattice Boltzmann simulations. Concluding remarks appear in Sec. $\mathbb{V}$, and detailed calculations of Green's functions, correlations and structure functions are contained in Appendix A.

\section{STEADY-STATE DENSITY FROM THE LINEAR RESPONSE APPROXIMA- TION}

To determine how weak spatial perturbations in the growth rate alter a steady-state density that is otherwise homogeneous and equal to the mean carrying capacity $\bar{K}=a_{0} / b$, we introduce the density deviation from the mean carrying capacity, normalized by the mean carrying capacity, as follows:

$$
\phi(\boldsymbol{x}) \equiv \frac{c^{*}(\boldsymbol{x})-\bar{K}}{\bar{K}}
$$

henceforth referred to as a (static) density fluctuation. Upon substituting the steady-state density of Eq. (的) into Eq. (四), we find that this (static) density fluctuation satisfies

$$
\left(-D \nabla^{2}+\mathbf{v} \cdot \nabla+a_{0}\right) \phi(\boldsymbol{x})=\delta a(\boldsymbol{x})+\left[\delta a(\boldsymbol{x}) \phi(\boldsymbol{x})-a_{0} \phi^{2}(\boldsymbol{x})\right]
$$

We now establish the linear response theory for the case of weak spatial fluctuations in the growth rate. First, we define $\varepsilon \equiv\left(\Delta / a_{0}\right) \geq\left|\delta a(\boldsymbol{x}) / a_{0}\right|$, a dimensionless measure of growth rate fluctuations. Observe that, in the absence of both advection and diffusion, non-zero populations at any point $\boldsymbol{x}$ will grow and saturate according to the local logistic growth process; the steady state density is then given by the local carrying capacity:

$$
c^{*}(\boldsymbol{x})=K(\boldsymbol{x}) \equiv a(\boldsymbol{x}) / b .
$$


Eq. (6), Eq. (8), and the bound associated with uniform distribution $\delta a(\boldsymbol{x}) \in[-\Delta, \Delta]$, implies that the density fluctuations obey $|\phi(\boldsymbol{x})| \lesssim \varepsilon$. In the presence of either diffusion or advection, populations traverse longer distances and sample a spatial average of local growth rates; the steady state density is then smoothed out, and the condition $|\phi(\boldsymbol{x})| \lesssim \varepsilon$ should remain approximately valid. In fact, when diffusion or advection become strong, numerical simulations reveal the suppression of density fluctuations, as shown for strong advection in Figs. [ and 6 in two dimensions and one dimension, respectively (the case of suppression by strong diffusion is similar.) Upon dividing Eq. (7) by $a_{0}$ and noting that $|\phi(\boldsymbol{x})|=O(\varepsilon)$, we see that the terms in the square bracket scale as $\varepsilon^{2}$, whereas the other terms scale as $\varepsilon$. Hence, in the limit of small $\varepsilon$, we can linearize Eq. (प):

$$
\left(-D \nabla^{2}+\mathbf{v} \cdot \nabla+a_{0}\right) \phi(\boldsymbol{x})=\delta a(\boldsymbol{x})
$$

Thus, in this linear approximation (used throughout this paper), static density fluctuations are generated in response to the growth disorder $\delta a(\boldsymbol{x})$ acting as a source term.

It is convenient to introduce the non-dimensionalized linear response equation to simplify further calculations. Upon defining the diffusion length in a growth time

$$
\xi_{D} \equiv \sqrt{D / a_{0}},
$$

and rescaled quantities

$$
\begin{aligned}
\tilde{\boldsymbol{x}} & \equiv \boldsymbol{x} / \xi_{D}, \\
\tilde{\mathrm{v}} & \equiv \mathrm{v} / 2 \sqrt{D a_{0}}=\mathrm{v} / \mathrm{v}_{F}, \\
U(\boldsymbol{x}) & \equiv \delta a(\boldsymbol{x}) / a_{0},
\end{aligned}
$$

Eq. (9) takes the dimensionless form

$$
\left(-\tilde{\nabla}^{2}+2 \tilde{\mathbf{v}} \cdot \tilde{\nabla}+1\right) \phi(\tilde{\boldsymbol{x}})=U(\tilde{\boldsymbol{x}}),
$$


where $\tilde{\nabla}$ denotes a gradient with respect to $\tilde{\boldsymbol{x}}$. The density fluctuations are then given by the convolution

$$
\phi(\tilde{\boldsymbol{x}})=\int G(\tilde{\boldsymbol{x}}-\tilde{\boldsymbol{y}}) U(\tilde{\boldsymbol{y}}) d^{d} \tilde{\boldsymbol{y}}
$$

where $G(\tilde{\boldsymbol{x}}-\tilde{\boldsymbol{y}})$ is a reaction-diffusion-advection Green's function that satisfies $\left(-\tilde{\nabla}^{2}+2 \tilde{\mathbf{v}} \cdot \tilde{\nabla}+1\right) G(\tilde{\boldsymbol{x}}-\tilde{\boldsymbol{y}}) \propto \delta^{d}(\tilde{\boldsymbol{x}}-\tilde{\boldsymbol{y}})$. The steady-state population density, corrected for diffusion and advection, then reads

$$
c^{*}(\tilde{\boldsymbol{x}})=\bar{K}\left[1+\int G(\tilde{\boldsymbol{x}}-\tilde{\boldsymbol{y}}) U(\tilde{\boldsymbol{y}}) d^{d} \tilde{\boldsymbol{y}}\right]
$$

And we must now determine $G(\tilde{\boldsymbol{x}}-\tilde{\boldsymbol{y}})$.

\section{A. Density modulation due to a point-like growth hot spot}

The Green's function of the reaction-diffusion-advection operator of Eq. (14) describes the response to a Dirac delta function source term, a point-like growth hot spot. In our rescaled coordinates, the Green's function satisfies

$$
\left(-\tilde{\nabla}^{2}+2 \tilde{\mathbf{v}} \cdot \tilde{\nabla}+1\right) G(\tilde{\boldsymbol{x}})=\frac{\Delta}{a_{0}} \delta^{d}(\tilde{\boldsymbol{x}})
$$

which can be simplified via the substitution

$$
G(\tilde{\boldsymbol{x}}) \equiv \exp \left(\tilde{\mathrm{v}} \tilde{x}_{\|}\right) G_{\tilde{\mathrm{v}}}(\tilde{\boldsymbol{x}})
$$

where $x_{\|}$is the direction along the advective flow. The result is a Helmholtz equation with the minus sign in the Laplacian:

$$
\left[-\tilde{\nabla}^{2}+\left(1+\tilde{\mathrm{v}}^{2}\right)\right] G_{\tilde{\mathrm{v}}}(\tilde{\boldsymbol{x}})=\frac{\Delta}{a_{0}} \delta^{d}(\tilde{\boldsymbol{x}})
$$


In $d$ dimensions, the isotropic solution of Eq. ([1.9), such that $G_{\tilde{\mathrm{v}}}(\tilde{\boldsymbol{x}})$ vanishes as $\tilde{x} \rightarrow \infty$, reads [4.9]

$$
\begin{aligned}
G_{\tilde{\mathrm{v}}}(\tilde{\boldsymbol{x}})= & \frac{\Delta}{a_{0}}\left(\frac{1}{\pi}\right)^{d / 2} \\
& \times\left[\left(\frac{|\tilde{\boldsymbol{x}}|}{\sqrt{1+\tilde{\mathrm{v}}^{2}}}\right)^{1-d / 2} K_{1-d / 2}\left(\sqrt{1+\tilde{\mathrm{v}}^{2}}|\tilde{\boldsymbol{x}}|\right)\right],
\end{aligned}
$$

where $K_{\alpha}(x)$ is the modified Bessel function of the second kind. Eqs. (20) and (18) are derived via direct Fourier transformation in Appendix $\mathbb{\text { Q }}$.

Eq. (188) encapsulates how advection breaks isotropy: by enhancing downstream response and suppressing upstream response in Eq. (20). Since $K_{\alpha}(x)=\sqrt{\frac{\pi}{2 x}} \exp (-x)\left[1+\frac{4 \alpha^{2}-1}{8 x}+\mathcal{O}\left(x^{-2}\right)\right]$ for large $x$ [50], Eq.(18) implies that $G(\tilde{\boldsymbol{x}})$ is exponentially localized in all transverse directions with the transverse localization length $\tilde{\xi}_{\perp}=1 / \sqrt{1+\tilde{\mathrm{v}}^{2}}$ similar to the localization length of $G_{\tilde{\mathrm{v}}}(\tilde{\boldsymbol{x}})$, i.e.,

$$
G\left(\tilde{x}_{\|}=0, \tilde{\boldsymbol{x}}_{\perp}\right) \sim \exp \left(-\sqrt{1+\tilde{\mathrm{v}}^{2}}\left|\tilde{\boldsymbol{x}}_{\perp}\right|\right)
$$

For $\tilde{\mathrm{v}} \gg 1, \tilde{\xi}_{\perp}$ contracts as $1 / \tilde{\mathrm{v}}$. However, the longitudinal localization lengths are asymmetric with an elongation in the downstream direction and a contraction in the upstream direction. For $\tilde{\mathrm{v}} \gg 1$,

$$
\begin{aligned}
G\left(\tilde{x}_{\|}, \tilde{\boldsymbol{x}}_{\perp}=\mathbf{0}\right) & \sim \exp \left(\tilde{\mathrm{v}} \tilde{x}_{\|}\right) \exp \left(-\sqrt{1+\tilde{\mathrm{v}}^{2}}\left|\tilde{x}_{\|}\right|\right) \\
& =\exp \left[\left(\tilde{x}_{\|}-\left|\tilde{x}_{\|}\right|\right) \tilde{\mathrm{v}}-\frac{\left|\tilde{x}_{\|}\right|}{2 \tilde{\mathrm{v}}}+O\left(\frac{1}{\tilde{\mathrm{v}}^{3}}\right)\right]
\end{aligned}
$$

thus, the downstream $\left(\tilde{x}_{\|}>0\right)$ and the upstream $\left(\tilde{x}_{\|}<0\right)$ localization lengths are given by $\tilde{\xi}_{\|}^{+} \approx 2 \tilde{\mathrm{v}}$ and $\tilde{\xi}_{\|}^{-} \approx 1 / 2 \tilde{\mathrm{v}}$, respectively. This steady state asymmetry, with a contraction in the transverse direction, differs from the early time growth dynamics, where diffusion spreads out the population superdiffusively in the transverse direction [19, 20, 23].

The steady-state response to advection is, however, constrained by a conservation law. By integrating Eq. ([7) over the whole domain with a periodic boundary condition, one finds that

$$
\int_{\Omega} G(\tilde{\boldsymbol{x}}) d^{d} \tilde{\boldsymbol{x}}=\frac{\Delta}{a_{0}}
$$

As a result, the overall response is suppressed at strong advection: the response amplitude 


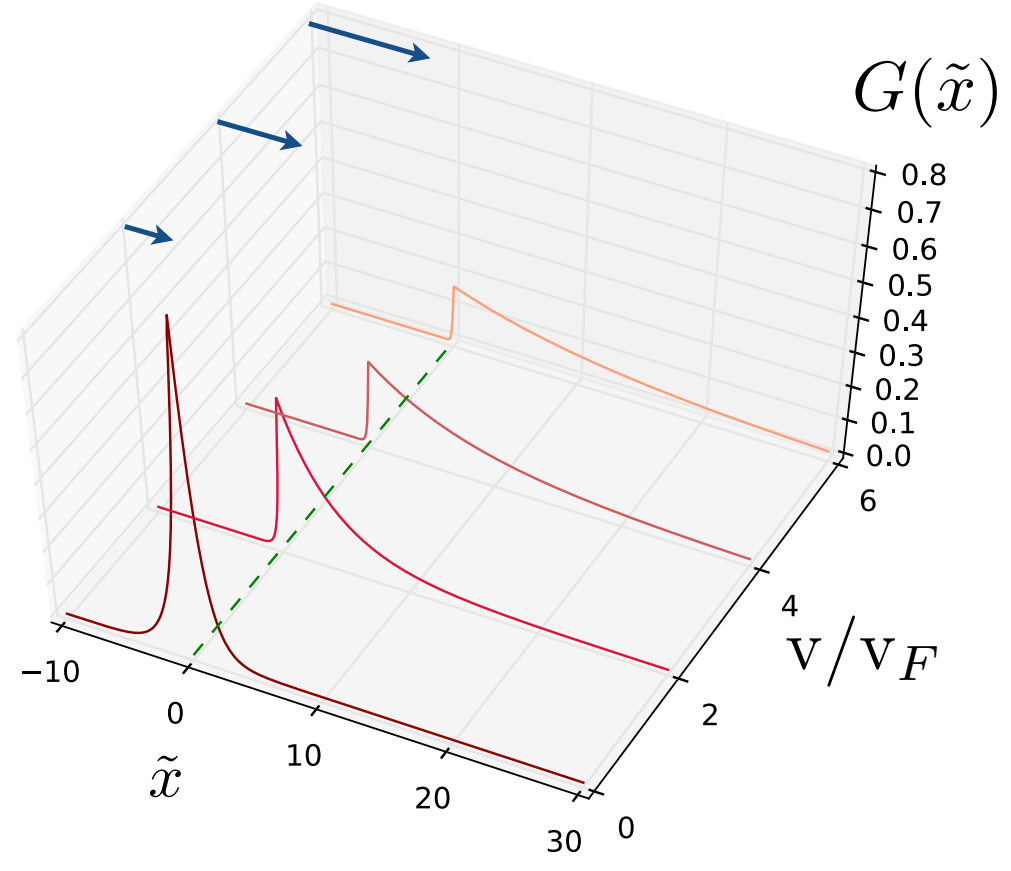

FIG. 2. (Color online) One-dimensional Green's functions given by Eq.(24) which describe the density fluctuations as a response to a point-like growth hot spot, introduced at the origin and indicated as the dashed green line. The response becomes strongly asymmetric as $\tilde{\mathrm{v}}$ increases, with the downstream localization length and the upstream localization length that scale respectively as $\tilde{\xi}_{\|}^{+}=2 \tilde{\mathrm{v}}$ and $\tilde{\xi}_{\|}^{-}=1 / 2 \tilde{\mathrm{v}}$ for $\tilde{\mathrm{v}} \gg 1$. Note that the response amplitude decays to compensate for the elongated downstream propagation. In this plot, the strength of the hot spot $\Delta / a_{0}$ is set to unity.

decreases to compensate for elongated downstream amplitude. Suppression of the response amplitude as a tradeoff for elongated downstream propagation is readily verified in onedimension. In this case, the modified Bessel function takes the simple form $K_{1 / 2}(x)=$ $\sqrt{\frac{\pi}{2 x}} \exp (-x)[50]$, and Eqs. (20)-(18) give

$$
G(\tilde{x})=\frac{\Delta}{a_{0}} \frac{1}{\sqrt{2}} \frac{\exp \left(\tilde{\mathrm{v}} \tilde{x}-\sqrt{1+\tilde{\mathrm{v}}^{2}}|\tilde{x}|\right)}{\sqrt{1+\tilde{\mathrm{v}}^{2}}} .
$$

Hence, advection leads to anisotropic response whose amplitude at $\tilde{x}=0$ decays as $1 / \sqrt{1+\tilde{\mathrm{v}}^{2}}$. Fig. 2. shows $G(\tilde{x})$ in one dimension for a variety of velocities. In two dimensions, Eqs. (20)-(18) give

$$
G\left(\tilde{x}_{\|}, \tilde{x}_{\perp}\right)=\frac{\Delta}{a_{0}}\left(\frac{1}{\pi}\right) \exp \left(\tilde{\mathrm{v}} \tilde{x}_{\|}\right) K_{0}\left(\sqrt{1+\tilde{\mathrm{v}}^{2}}|\tilde{\boldsymbol{x}}|\right)
$$


(a) $\quad \mathrm{v}=0$
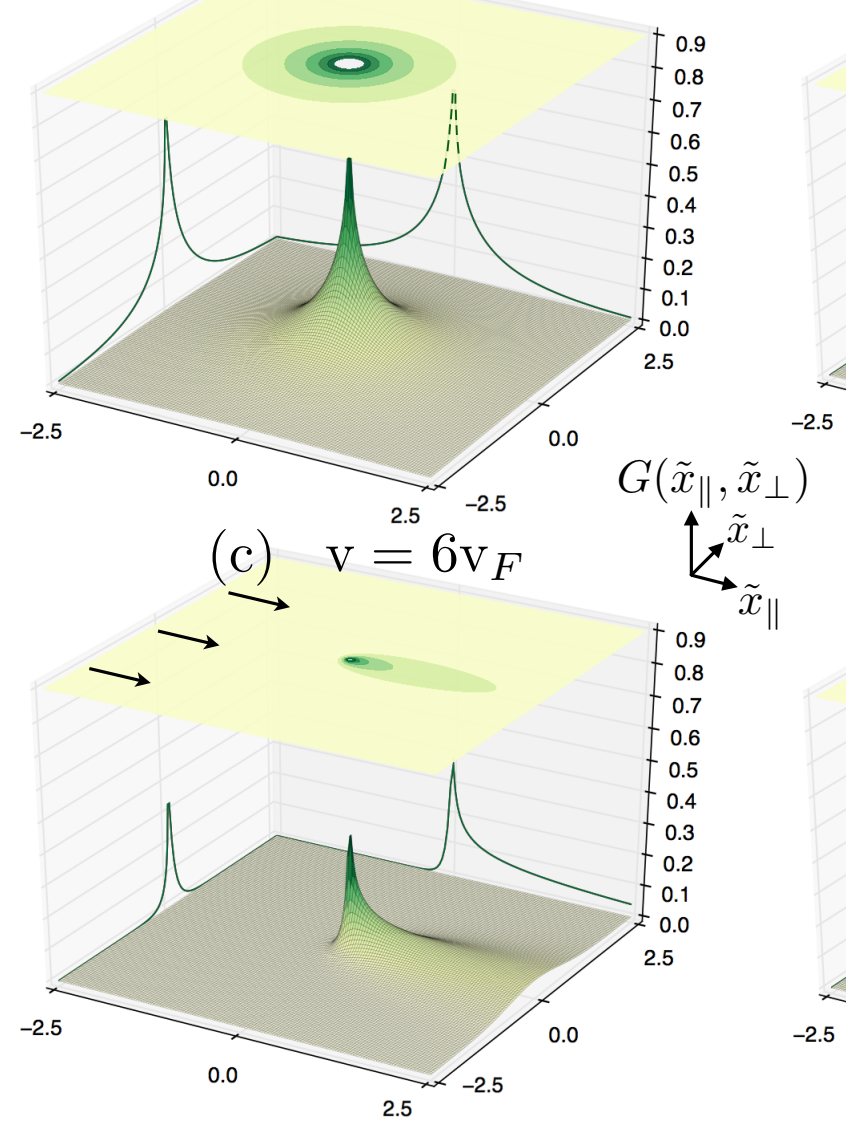

(b) $\mathrm{v}=3 \mathrm{v}_{F}$
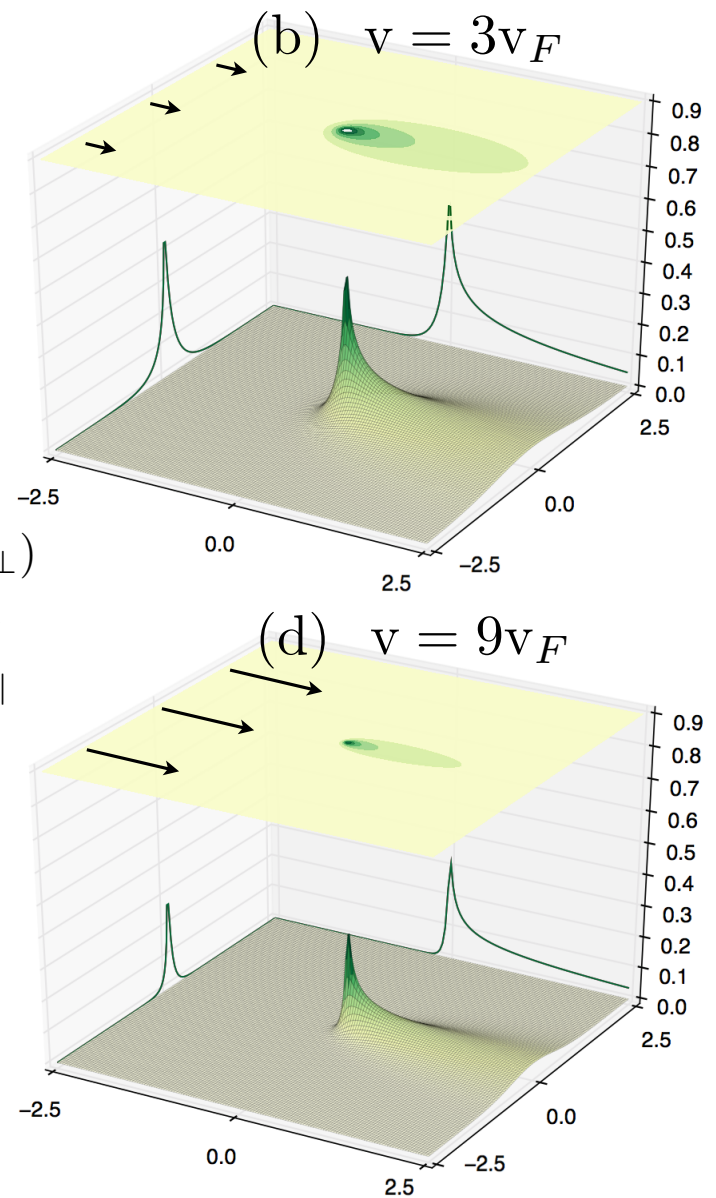

FIG. 3. (Color online) Two-dimensional Green's function of Eq.(25)). The response to a pointlike growth hot spot is isotropic in the absence of flow, as represented in (a), but streaked out in the direction parallel to the flow with an elongated downstream localization length, as shown in (b)-(d). With increasing flow speed, the overall response amplitude decays. In addition, the transverse response, controlled by $G_{\tilde{\mathrm{v}}}(\tilde{\boldsymbol{x}}) \sim K_{0}\left(\sqrt{1+\tilde{\mathrm{v}}^{2}}|\tilde{\boldsymbol{x}}|\right)$, contracts symmetrically, decaying exponentially with the localization length $\xi=1 / \sqrt{1+\tilde{\mathrm{v}}^{2}}$. Similar to one dimension, $\tilde{\xi}_{\|}^{+}=2 \tilde{\mathrm{v}}$ and $\tilde{\xi}_{\|}^{-}=1 / 2 \tilde{\mathrm{v}}$ in the flow direction for $\tilde{\mathrm{v}} \gg 1$. Solid lines shown on the plane $\tilde{x}_{\|}=-2.5$ and $\tilde{x}_{\perp}=2.5$ are cross-sections of the response along the plane $\tilde{x}_{\|}=0$ and the plane $\tilde{x}_{\perp}=0$, respectively. These cross-sections reveal the behavior of localization lengths at different velocities. The boundaries of the colored contour above each plot correspond to the Green's function contours $0.0,0.05,0.1,0.15,0.2,0.25$ and 0.3 , with the color ranging from yellow to dark green, respectively. The reduction in the area of these colored contours at stronger advection reflects the decay of the response amplitude. In this plot, the amplitude of the $\delta$-function hot spot $\Delta / a_{0}$ is set to 1 .

Although there is a logarithmic singularity at $|\tilde{\boldsymbol{x}}|=0$ (cut off by, say, the spacing between microorganisms), one can see from the profiles of the plots of Eq. (25) in Fig. 3 that the overall response amplitude is again suppressed at strong advection. Fig. 3 also illustrates the process of symmetric transverse contraction, in addition to the asymmetric downstream 
elongation and upstream contraction in the longitudinal direction.

\section{LONGITUDINAL STRIATIONS IN THE STEADY STATE FOR STRONG} ADVECTION IN $d \geq 2$

Although the linear response theory developed in Sec. 미 suggests that the transverse localization length of an isolated growth hot spot shrinks to zero as the advection speed increases, we now show that superposition of the responses from uncorrelated growth disorder leads to correlations whose transverse correlation length approaches a finite velocityindependent value, while the longitudinal correlation length grows linearly with $\tilde{\mathrm{v}}$. The result is striated population correlations in the steady state.
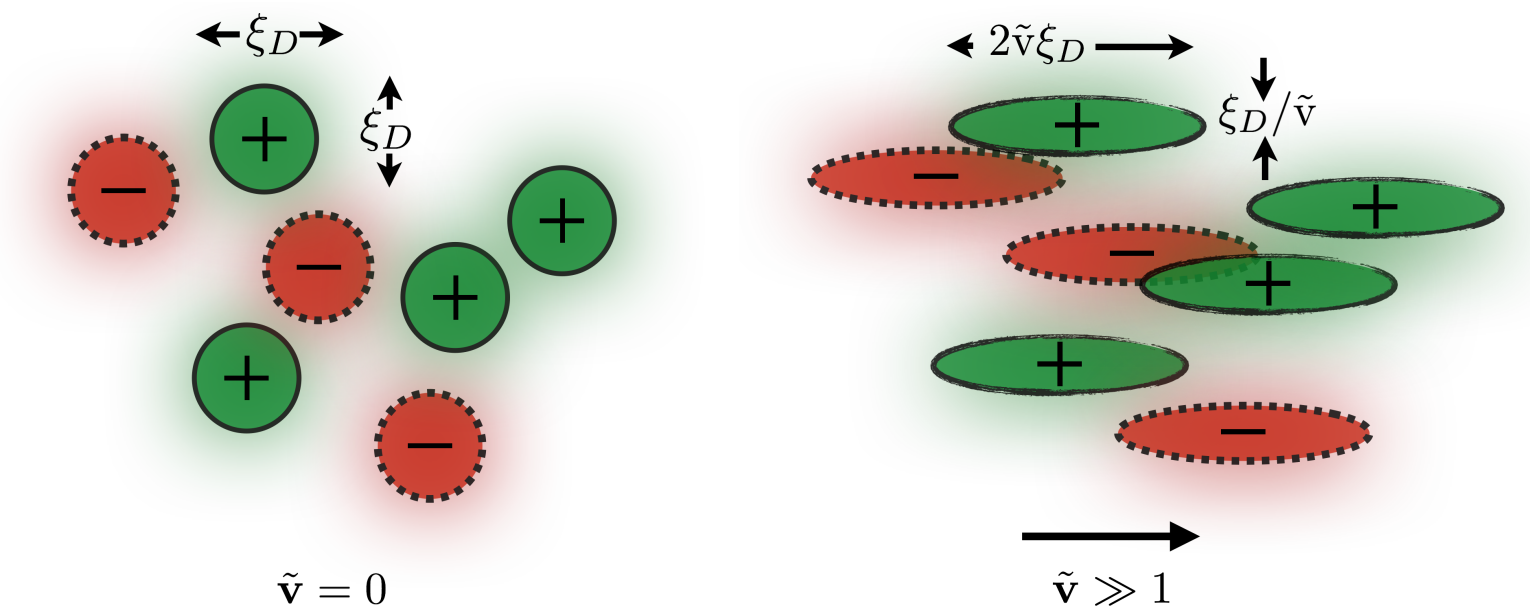

FIG. 4. (Color online) How striations driven by flow and spatially quenched growth disorder arise in two dimensions. (Left) Without advection, density fluctuations arise from the superposition of the isotropic responses $G_{\mathrm{v}=0}\left(\boldsymbol{x}-\boldsymbol{x}^{\prime}\right)$ centered around a source $\left(U\left(\boldsymbol{x}^{\prime}\right)>0\right)$ or a $\operatorname{sink}\left(U\left(\boldsymbol{x}^{\prime}\right)<0\right)$, depicted in green and red respectively. The localization length is given by $\xi_{D}=\sqrt{D / a_{0}}$. (Right) For strong advection $\tilde{\mathrm{v}} \gg 1$, each response is distorted asymmetrically: the downstream localization length grows as $\xi_{\|}^{+}=2 \tilde{\mathrm{v}} \xi_{D}$, and the transverse localization length contracts as $\xi_{\perp}=\xi_{D} / \tilde{\mathrm{v}}$. Although each response is subjected to transverse contraction that shrinks to zero at stronger flow, the random superposition of responses exhibit non-vanishing transverse correlations, as shown in Figs. 5 and 8 .

\section{A. Two-point correlation function and the striation pattern}

To model rapid, statistically isotropic spatial variations in the growth rates, we assume each local growth rate $U(\tilde{\boldsymbol{x}})=\delta a(\tilde{\boldsymbol{x}}) / a_{0}$ is drawn from a uniform box distribution in the 
interval $\left[-\Delta / a_{0}, \Delta / a_{0}\right]$, which gives the two-point noise correlation function

$$
\left\langle U(\tilde{\boldsymbol{x}}) U\left(\tilde{\boldsymbol{x}}^{\prime}\right)\right\rangle=\frac{1}{3} \frac{\Delta^{2}}{a_{0}^{2}} \delta^{d}\left(\tilde{\boldsymbol{x}}-\tilde{\boldsymbol{x}}^{\prime}\right)
$$

where $\langle\cdot\rangle$ denotes ensemble averages over disordered growth rate realizations. In the Fourier domain, Eq.(26) gives white noise with magnitude

$$
\left\langle|U(\tilde{\boldsymbol{q}})|^{2}\right\rangle=\frac{1}{3} \frac{\Delta^{2}}{a_{0}^{2}}
$$

We expect statistical translational invariance, $\langle\phi(\tilde{\boldsymbol{x}}) \phi(\mathbf{0})\rangle \equiv\left\langle\phi\left(\tilde{\boldsymbol{x}}+\tilde{\boldsymbol{x}}^{\prime}\right) \phi\left(\tilde{\boldsymbol{x}}^{\prime}\right)\right\rangle$, so the two-point correlation function of the density fluctuations is given by

$$
\langle\phi(\tilde{\boldsymbol{x}}) \phi(\mathbf{0})\rangle=\int \frac{d^{d} \tilde{q}}{(2 \pi)^{d}} e^{i \tilde{\boldsymbol{q}} \cdot \tilde{\boldsymbol{x}}} S(\tilde{\boldsymbol{q}})
$$

where the static structure factor in the steady state is related to the Fourier transformed Green's function $G(\tilde{\boldsymbol{q}})$,

$$
\begin{aligned}
S(\tilde{\boldsymbol{q}}) & \equiv\left\langle|\phi(\tilde{\boldsymbol{q}})|^{2}\right\rangle \\
& =\left\langle|U(\tilde{\boldsymbol{q}})|^{2}\right\rangle|G(\tilde{\boldsymbol{q}})|^{2} \\
& =\frac{\left\langle|U(\tilde{\boldsymbol{q}})|^{2}\right\rangle}{\left[\left(\tilde{q}^{2}+1\right)^{2}+4 \tilde{\mathrm{v}}^{2} \tilde{q}_{\|}^{2}\right]} .
\end{aligned}
$$

Upon substituting Eqs. (29) and (27) into Eq. (28), the two-point correlation function of the density fluctuations with spatially uncorrelated random growth rates reads

$$
\langle\phi(\tilde{\boldsymbol{x}}) \phi(\mathbf{0})\rangle=\frac{\Delta^{2}}{3 a_{0}^{2}} \int \frac{d^{d} \tilde{q}}{(2 \pi)^{d}} \frac{e^{i \tilde{\boldsymbol{q}} \cdot \tilde{\boldsymbol{x}}}}{\left[\left(\tilde{q}^{2}+1\right)^{2}+4 \tilde{\mathrm{v}}^{2} \tilde{q}_{\|}^{2}\right]} .
$$

We now evaluate Eq. (30) in various dimensions, with details relegated to Appendix $\mathrm{A}$. For $d=1$, Eq. (30) can be evaluated via contour integration, with the result ( $\operatorname{setting} \tilde{x}_{\|}=\tilde{x}$ )

$$
\begin{aligned}
\langle\phi(\tilde{x}) \phi(0)\rangle & =\frac{\Delta^{2}}{24 a_{0}^{2}} \frac{1}{\tilde{\mathrm{v}} \sqrt{1+\tilde{\mathrm{v}}^{2}}} \\
& \times\left[\frac{1}{g_{-}(\tilde{\mathrm{v}})} e^{-g_{-}(\tilde{\mathrm{v}})|\tilde{x}|}-\frac{1}{g_{+}(\tilde{\mathrm{v}})} e^{-g_{+}(\tilde{\mathrm{v}})|\tilde{x}|}\right],
\end{aligned}
$$


where the two exponential decays are controlled by $g_{ \pm}(\tilde{\mathrm{v}}) \equiv \sqrt{1+\tilde{\mathrm{v}}^{2}} \pm \tilde{\mathrm{v}}$. Although this result appears singular at $\tilde{\mathrm{v}}=0$, the limit $\tilde{\mathrm{v}} \rightarrow 0$ is in fact well-defined, and given by

$$
\lim _{\tilde{\mathrm{v}} \rightarrow 0}\langle\phi(\tilde{x}) \phi(0)\rangle=\frac{\Delta^{2}}{12 a_{0}^{2}}(1+|\tilde{x}|) e^{-|\tilde{x}|}
$$

The exponential localization associated with a single hot spot is broadened by a factor $(1+|\tilde{x}|)$; the correlation length, however, is the same as the diffusive localization length $\xi_{D}$. For strong advection, $g_{-}(\tilde{\mathrm{v}})=2 \tilde{\mathrm{v}}+1 / 2 \tilde{\mathrm{v}}+O\left(1 / \tilde{\mathrm{v}}^{3}\right)$ and $g_{+}(\tilde{\mathrm{v}})=1 / 2 \tilde{\mathrm{v}}+O\left(1 / \tilde{\mathrm{v}}^{3}\right)$; the first term in the square bracket of Eq. (31) dominates, resulting in

$$
\lim _{\tilde{\mathrm{v}} \rightarrow \infty}\left\langle\phi\left(\tilde{x}_{\|}\right) \phi(0)\right\rangle \sim \frac{e^{-\left|\tilde{x}_{\|}\right| / 2 \tilde{\mathrm{v}}}}{\tilde{\mathrm{v}}} .
$$

For $d=2$, the spatial structure of steady state is embodied in the longitudinal correlation function $\left\langle\phi\left(\tilde{x}_{\|}, 0\right) \phi(\mathbf{0})\right\rangle$ and the transverse correlation function $\left\langle\phi\left(0, \tilde{x}_{\perp}\right) \phi(\mathbf{0})\right\rangle$ that are defined by Eq. (30). In the absence of advection, the correlation function is isotropic and is given by

$$
\lim _{\tilde{v} \rightarrow 0}\langle\phi(\tilde{\boldsymbol{x}}) \phi(\mathbf{0})\rangle \sim|\tilde{\boldsymbol{x}}| K_{1}(|\tilde{\boldsymbol{x}}|)
$$

where $K_{1}(x)$ is the modified Bessel function of a second kind that decays exponentially at large distance $x$ as $K_{1}(x)=\sqrt{\frac{\pi}{2 x}} \exp (-x)\left[1+3 / 8 x+\mathcal{O}\left(x^{-2}\right)\right]$ for $x \gg 1$ [50]]. The correlation length is thus given by diffusive correlation length $\xi_{D}$ in this limit. In the strong advection limit, however, correlations become highly anisotropic:

$$
\begin{aligned}
\lim _{\tilde{\mathrm{v}} \rightarrow \infty}\left\langle\phi\left(\tilde{x}_{\|}, 0\right) \phi(\mathbf{0})\right\rangle & \sim\left(\frac{\left|\tilde{x}_{\|}\right|}{2 \tilde{\mathrm{v}}}\right)^{1 / 4} K_{-1 / 4}\left(\frac{\left|\tilde{x}_{\|}\right|}{2 \tilde{\mathrm{v}}}\right), \\
\lim _{\tilde{\mathrm{v}} \rightarrow \infty}\left\langle\phi\left(0, \tilde{x}_{\perp}\right) \phi(\mathbf{0})\right\rangle & \sim \frac{e^{-\left|\tilde{x}_{\perp}\right|}}{\tilde{\mathrm{v}}}
\end{aligned}
$$

with the longitudinal and transverse correlation lengths that behave as follows,

$$
\begin{aligned}
& \lim _{\tilde{\mathrm{v}} \rightarrow \infty} \xi_{\|}=\xi_{\mathrm{v}}=\frac{\mathrm{v}}{a_{0}}, \\
& \lim _{\tilde{\mathrm{v}} \rightarrow \infty} \xi_{\perp}=\xi_{D}=\sqrt{\frac{D}{a_{0}}} .
\end{aligned}
$$

Note the limiting transverse correlation length is given by a velocity-independent, diffusive 
localization length. However, the ratio of transverse to longitudinal correlation lengths becomes highly anisotropic:

$$
\lim _{\tilde{\mathrm{v}} \rightarrow \infty}\left(\frac{\xi_{\|}}{\xi_{\perp}}\right)=\frac{\mathrm{v}}{\sqrt{D a_{0}}}=2 \tilde{\mathrm{v}}
$$

These anisotropic patterns might arise in natural marine microbial populations, their Fisher wave spreading velocity is much smaller than oceanic flow speed; for a motile bacteria, a typical diffusion constant $D$ is of order $10^{-5} \mathrm{~cm}^{2} \mathrm{~s}^{-1}$ and the typical doubling time $a_{0}$ is of order $10^{-3} \mathrm{~s}^{-1}$ which gives $\mathrm{v}_{F}=2 \sqrt{D a_{0}}$ of order $1 \mu \mathrm{m} \mathrm{s}^{-1}$ [3.] , which is indeed small compared to a typical oceanic current; see Ref. [30] and references therein.

Note that uncorrelated disorder (viewed as a superposition of responses from growth hot spots) leads to a non-zero transverse correlation length, as shown in Eq. (38) for two dimensions, in contrast to the result for a single point-like growth hot-spot of Sec. एА. In fact, a finite transverse correlation arises for all $d \geq 2$. To see this, consider the transverse correlation functions:

$$
\begin{aligned}
\left\langle\phi\left(0, \tilde{\boldsymbol{x}}_{\perp}\right) \phi(\mathbf{0})\right\rangle & \sim \int \frac{d^{d-1} \tilde{q}_{\perp}}{(2 \pi)^{d-1}} e^{i \tilde{\boldsymbol{q}}_{\perp} \cdot \tilde{\boldsymbol{x}}_{\perp}} \int \frac{d \tilde{q}_{\|}}{2 \pi} \frac{1}{\left[\left(\tilde{q}^{2}+1\right)^{2}+4 \tilde{\mathrm{v}}^{2} \tilde{q}_{\|}^{2}\right]} \\
& =\pi \int \frac{d^{d-1} \tilde{q}_{\perp}}{(2 \pi)^{d}} \frac{e^{i \tilde{\boldsymbol{q}}_{\perp} \cdot \tilde{\boldsymbol{x}}_{\perp}}}{\left(\tilde{q}_{\perp}^{2}+1\right)\left(\tilde{q}_{\perp}^{2}+1+\tilde{\mathrm{v}}^{2}\right)^{1 / 2}} .
\end{aligned}
$$

Upon taking the limit $\tilde{\mathrm{v}} \gg 1$, it follows that

$$
\begin{aligned}
\lim _{\tilde{\mathrm{v}} \rightarrow \infty}\left\langle\phi\left(0, \tilde{\boldsymbol{x}}_{\perp}\right) \phi(\mathbf{0})\right\rangle & \sim \frac{\pi}{\tilde{\mathrm{V}}} \int \frac{d^{d-1} \tilde{q}_{\perp}}{(2 \pi)^{d}} \frac{e^{i \tilde{\boldsymbol{q}}_{\perp} \cdot \tilde{\boldsymbol{x}}_{\perp}}}{\left(\tilde{q}_{\perp}^{2}+1\right)}, \\
& =\frac{e^{-\left|\tilde{x}_{\perp}\right|}}{4 \tilde{\mathrm{v}}},
\end{aligned}
$$

which show that the transverse correlation length still obeys Eq. (38) for $d \geq 2$. Thus, the limiting transverse correlations in higher dimensions remains velocity-independent and is characterized by the diffusive localization length $\xi_{D}$, while the amplitude of fluctuations decays at increasing advection as $\lim _{\tilde{\mathrm{v}} \rightarrow \infty}\left\langle\phi^{2}(\mathbf{0})\right\rangle \sim 1 / \tilde{\mathrm{v}}$. 


\section{B. Comparisons to numerical simulations}

We now check theoretical predictions in Sec. WA by comparing the theoretical structure factor $S_{\mathrm{th}}(\tilde{\boldsymbol{q}})$ given by Eq. (2.9) to the structure factor $S_{\text {num }}(\tilde{\boldsymbol{q}})$ calculated from the numerical solution of Eq. (团). To obtain the numerical steady state satisfying Eq. (团), we inoculate the simulation domain with a uniform concentration equal to the mean carrying capacity $\bar{K}$, and numerically evolve the time-dependent advective FKPP Eq. (3) until the steady state is reached using the Lattice Boltzmann method, a robust lattice discretization scheme for advective and diffusive transports [51, 52] that can incorporate reactive agents [53]. This method achieves high numerical accuracy for reaction-diffusion-advection problems, even in the strong advection limit [54]. Random growth rate $U\left(\tilde{\boldsymbol{x}}_{i}\right)=\delta a\left(\tilde{\boldsymbol{x}}_{i}\right) / a_{0}$ is introduced on each lattice site $\tilde{\boldsymbol{x}}_{i}$, and is independently drawn from a uniform box distribution in the interval $\left[-\Delta / a_{0}, \Delta / a_{0}\right]$. The 9-speed 2-dimensional (D2Q9) lattice is adopted to evolve the density field according to Eq. (3) on a two-dimensional square lattice with $N_{x} \times N_{y}$ sites and a periodic boundary condition implemented by adding the buffer sites at the boundaries [53]. In this scheme, time evolution on a one-dimensional lattice with a periodic boundary condition also follows immediately, provided $N_{x}$ is set to 1 . Once the numerical steady state $c_{\mathrm{num}}^{*}(\boldsymbol{x})$ is reached, one can calculate the squared modulus of the discrete fourier transform of the steady state density fluctuations, denoted by $\left|\phi_{\text {num }}(\tilde{\boldsymbol{q}})\right|^{2}$. After taking ensemble averages over the random growth rates, this results in the numerical structure factor $S_{\text {num }}(\tilde{\boldsymbol{q}})$. This numerical structure factor is the discrete counterpart of the theoretical structure factor $S_{\mathrm{th}}(\tilde{\boldsymbol{q}})$ predicted by Eq. (29)).

Figures 6 and 10 report numerical steady states obtained from the Lattice Boltzmann simulations with random growth rates in one and two dimensions. These simulations show the decrease in the amplitude of the density fluctuations as advection becomes stronger, confirming the assumption of the linear response theory in Sec. ㅁ. In addition, unlike standard finite-difference schemes in which advection can lead to spurious alignment of population structure along lattice directions [20], the D2Q9 discretization, which ensures the fourth order isotropy of lattice tensors, avoids the artifact of preferred lattice orientations. The left column of Fig. 5 illustrates the steady state density fluctuations for the same realization of the random growth rate for different advection velocities: $\mathbf{v}=\mathbf{0}$ in Fig. $5(\mathrm{a}), \mathrm{v}=8 \mathrm{v}_{F}$ along the $x$-axis in Fig. $5(\mathrm{c})$, and $\mathrm{v}=8 \mathrm{v}_{F}$ inclined at $37^{\circ}$ relative to the directions associ- 

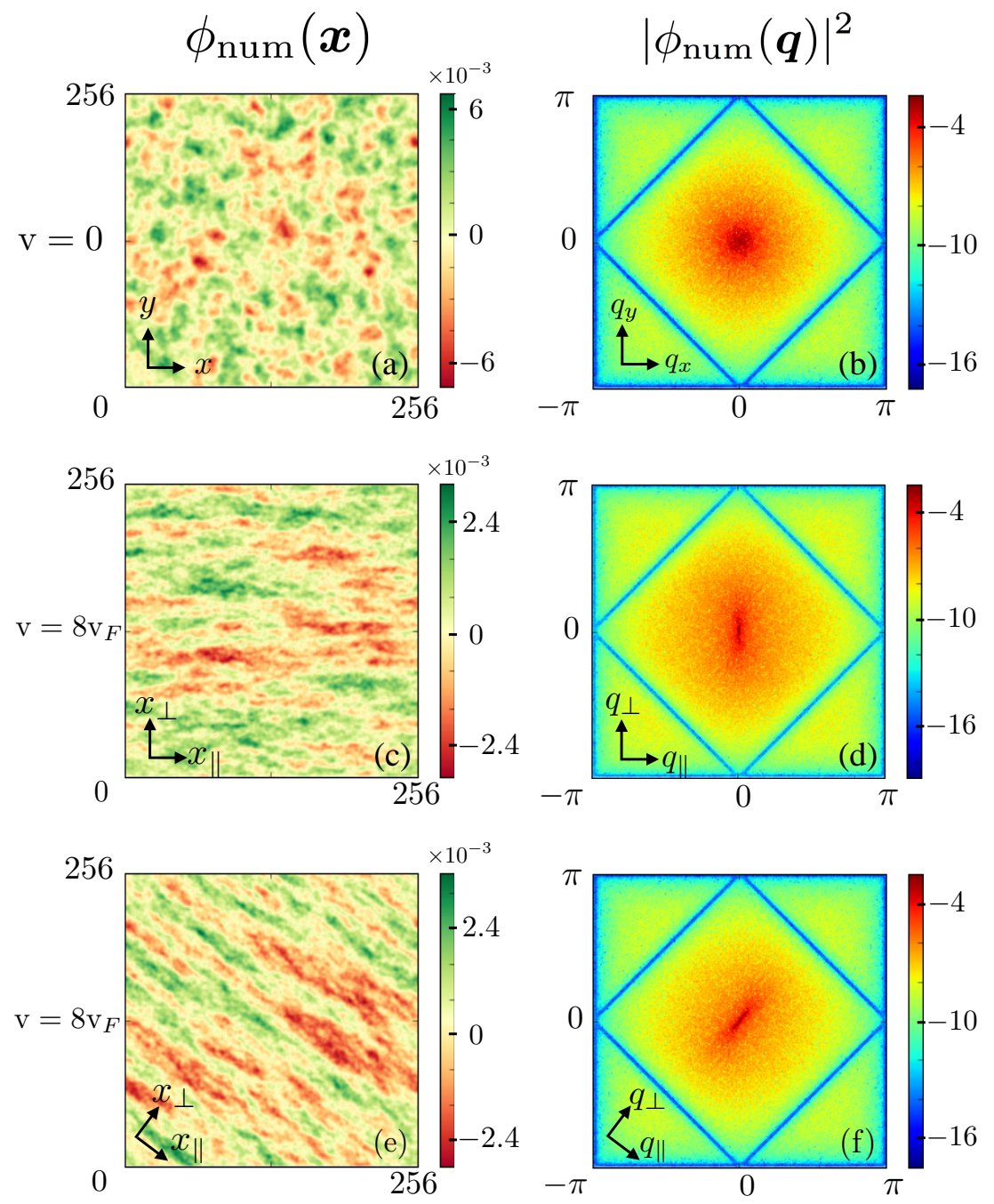

FIG. 5. (Color online) Steady state density fluctuations $\phi_{\text {num }}(\boldsymbol{x}) \equiv\left[c_{\text {num }}^{*}(\boldsymbol{x})-\bar{K}\right] / \bar{K}$ from Lattice Boltzmann simulations with $256^{2}$ sites (left) and the squared modulus of the discrete fourier transform of the steady state density fluctuations $\left|\phi_{\text {num }}(\boldsymbol{q})\right|^{2}$ (right) for the same disorder realization but different flow velocities: $\mathrm{v}=0$ in (a) and (b), $\mathrm{v}=8 \mathrm{v}_{F}$ along the $x$-axis in (c) and (d), and $\mathrm{v}=8 \mathrm{v}_{F}$ inclined at a $37^{\circ}$ angle relative to the periodic boundary conditions in (e) and (f). The coordinate $\boldsymbol{x}=(x, y)$ and the wavevector $\boldsymbol{q}=\left(q_{x}, q_{y}\right)$ are displayed in the original (dimensional) scale with the lattice unit $l_{0}$ set to 1 . The color codes for the structure factors ((b), (d), and (f)) are displayed in base-10 logarithmic scale. The parameters in this figure are $D=5 \times 10^{-4}, a_{0}=10^{-5}, \Delta=0.1 a_{0}$, and $b=10^{-9}$, which give $\xi_{D}=\sqrt{D / a_{0}} \approx 7$, $\mathrm{v}_{F}=2 \sqrt{D a_{0}} \approx 1.4 \times 10^{-4}$, and $\bar{K}=a_{0} / b=10^{4}$. Long wavelength modes in the absence of advection, in (b), appear statistically isotropic as expected. The blue diamonds at large $q$ in (b), (d), and (f) are an artifact of the underlying lattice.

ated with the periodic boundary conditions in Fig. 5(d). The steady state populations in 
Figs. 5(c) and (e) streak out along the advection direction, despite the bias imposed by an underlying square lattice. The elongation of steady state populations along the advection direction also appears in the Fourier-transformed $\left|\phi_{\text {num }}(\boldsymbol{q})\right|^{2}$ shown on the right column of Fig. 5. There, the short wavelength modes in the longitudinal direction are noticeably suppressed, while the modes in the transverse direction are only mildly modified, leading to elliptical contours at small $q$ 's with the minor and major axis aligned along the longitudinal and transverse direction, respectively. Notice the plots of $\left|\phi_{\text {num }}(\boldsymbol{q})\right|^{2}$ are approximately zero whenever $\boldsymbol{q} \cdot \boldsymbol{r}_{\text {lat }}= \pm \pi / l_{0}= \pm \pi$, where $\boldsymbol{r}_{\text {lat }}$ belongs to either the nearest neighbor basis $\left\{\boldsymbol{e}_{x}, \boldsymbol{e}_{y}\right\}$ or the next nearest neighbor basis $\left\{\boldsymbol{e}_{x}+\boldsymbol{e}_{y}, \boldsymbol{e}_{x}-\boldsymbol{e}_{y}\right\}$ of the square lattice, corresponding to the blue squares or the blue diamonds, respectively. These short-wavelength anisotropic lattice artifacts arise from the D2Q9 scheme that allows both nearest and next nearest neighbor hopping on the square lattice. However, the long-wavelength modes ( $q$ near the origin), which characterize the macroscopic striations, are further away from the blue diamond and are orders of magnitude larger than zone-boundary modes with wavenumber $q \gtrsim \pi / \sqrt{2} l_{0}=\pi / \sqrt{2}$. As discussed above, the long-wavelength physics is insensitive to these lattice artifacts, as can be confirmed by rotating Fig. 5(f) counterclockwise by an angle $37^{\circ}$ and comparing to Fig. 5(d). We thus expect that the long-range striation patterns are well-described by the behavior of $S_{\text {num }}(\tilde{\boldsymbol{q}})$ near the origin, as determined by the Lattice Boltzmann simulations, averaged over ensembles of growth rates.

We now compare the long-wavelength modes of $S_{\text {num }}(\tilde{\boldsymbol{q}})$ to $S_{\mathrm{th}}(\tilde{\boldsymbol{q}})$ predicted by Eq. (2.9). Although theoretical results of Sec. एШA assume spatially uncorrelated growth disorder whereas the Lattice Boltzmann simulation has an intrinsic short-range disorder correlation on the order of the lattice size $l_{0} \equiv 1$, we expect that the long-distance statistics are insensitive to such microscopic details, provided the correlation length of the emerging pattern is larger than the lattice size. Because advection stretches out correlations in the longitudinal direction, the approximation to a continuum model should become even more accurate at stronger advection. In fact, as shown in the comparisons between $S_{\text {num }}(\tilde{\boldsymbol{q}})$ to $S_{\mathrm{th}}(\tilde{\boldsymbol{q}})$ in one and two dimensions in Figs 7 and 8 respectively, excellent agreement is obtained at long wavelengths even when $\xi_{D}$ is of order $2 l_{0}=2$, which is only twice the correlation length of the simulated noise. The agreement is even better for stronger advection. Thus, the uncorrelated noise predictions of Sec. ШШA are able to capture the long-range statistics of the striation pattern, despite the presence of inherent lattice-scale correlations of the simulated 
noise.

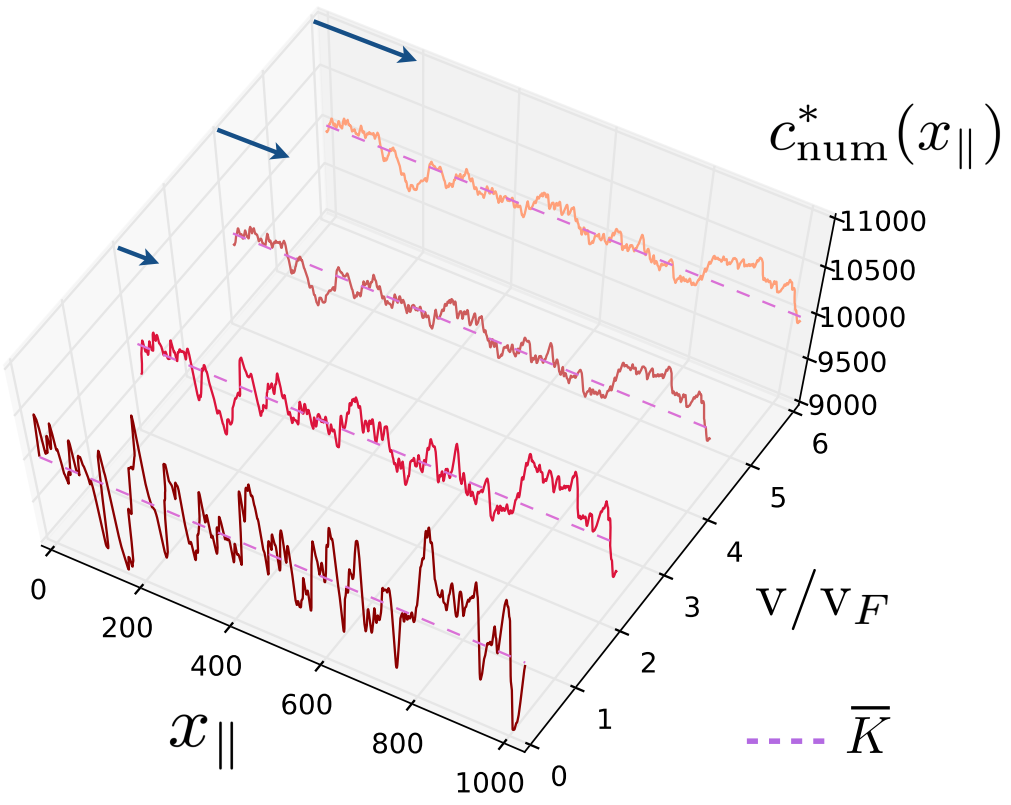

FIG. 6. Typical steady-state densities $c_{\text {num }}^{*}\left(x_{\|}\right)$in one dimension due to growth disorder and advection from the lattice Boltzmann simulation using a $D 2 Q 9$ scheme of size $1 \times 1024$ sites. The parameters are $D=5 \times 10^{-4}, a_{0}=1 \times 10^{-5}, \Delta=0.4 a_{0}$, and $b=10^{-9}$, resulting in $\xi_{D}=$ $\sqrt{D / a_{0}} \approx 7.07, \mathrm{v}_{F}=2 \sqrt{D a_{0}} \approx 1.4 \times 10^{-4}$, and $\bar{K}=a_{0} / b=10^{4}$. The dashed lines denote the local carrying capacity $\bar{K}$. As advection becomes stronger, density fluctuations are suppressed and (longitudinal) correlation lengths stretch out. The details of correlations in one dimensional systems are elucidated in the structure factors of Fig.7.

\section{CONCLUSIONS AND OUTLOOK}

Previous work has demonstrated the important role of spatially quenched disorder in the local growth rates on the long-time population structure that disperses through unidirectional advection and diffusion in hostile growth environments [21, 23, 24, 34]. Here, we study the role of growth disorder on the long-time population structure of populations in fertile random growth environments in which the local carrying capacity is strictly positive with weak disorder in the growth rates. To leading order in the strength of weak growth disorder, fluctuations from the homogeneous carrying capacity of steady-state populations can be perturbatively treated as a response to growth disorder.

Within the linear response theory, growth disorder and strong fluid advection leads to the emergence of striated steady-state population structure in two or higher dimensions 


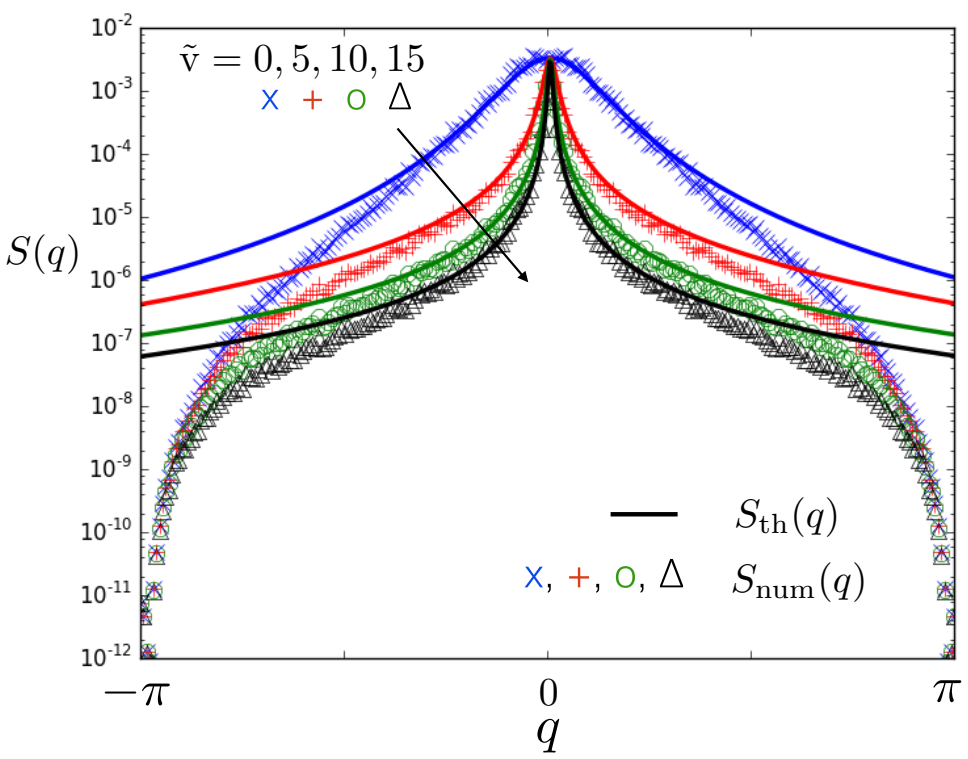

FIG. 7. (Color online) Comparisons between the predicted one-dimensional structure factor $S_{\text {th }}(q)$ (solid lines), and numerical structure factor $S_{\text {num }}(q)$ (symbols) from the lattice Boltzmann simulation with $1 \times 256$ sites. The wavenumber $q$ is displayed in the original (dimensional) scale with the lattice unit $l_{0}$ set to 1 . Simulation results are obtained from averaging the structure factors of density fluctuations over 200 growth rate realizations. The predicted long-wavelength modes of $S_{\text {th }}(q)$ are in excellent agreement with those of the simulated $S_{\text {num }}(q)$. Although the simulated structure factors are affected by inherent lattice-size disorder correlations near $q \sim \pi / l_{0}=\pi$, the $q \rightarrow 0$ modes are much larger (note the logarithmic scale on the $S(q)$ axis). At strong advection when $\tilde{\mathrm{v}} \gg 1$, long-wavelength modes are even more pronounced, and $S_{\mathrm{th}}(q)$ is even closer to $S_{\text {num }}(q)$. The parameters in this figure are $D=5 \times 10^{-4}, a_{0}=9 \times 10^{-5}, \Delta=0.1 a_{0}$, and $b=10^{-9}$, giving $\xi_{D}=\sqrt{D / a_{0}} \approx 2.36, \mathrm{v}_{F}=2 \sqrt{D a_{0}} \approx 4.2 \times 10^{-4}$, and $\bar{K}=a_{0} / b=9 \times 10^{4}$.

with a periodic boundary condition. For large advection speeds $\mathrm{v} \gg \mathrm{v}_{F}=2 \sqrt{D a_{0}}$, the longitudinal correlation length elongates as $\mathrm{v} / a_{0}$ and the transverse correlation length approaches the finite velocity-independent value given by the diffusive localization length $\xi_{D}=\sqrt{D / a_{0}}$. In contrast to steady-state populations with a point-like growth hot spot, whose transverse localization length contracts to zero as the advection speed increases, spatially quenched random growth disorder impedes the disappearance of transverse correlations. In our case, transverse correlations exhibit anomalous transverse diffusive spreading behavior of the early-time growth dynamics at strong advection as studied in Ref. [23]. The Lattice Boltzmann simulation of the generalized FKPP equation (3) confirms the emergence of striated population structure at strong advection, and verifies the predicted long-distance statistics from the linear response theory in one and two dimensions. Similar striated popu- 

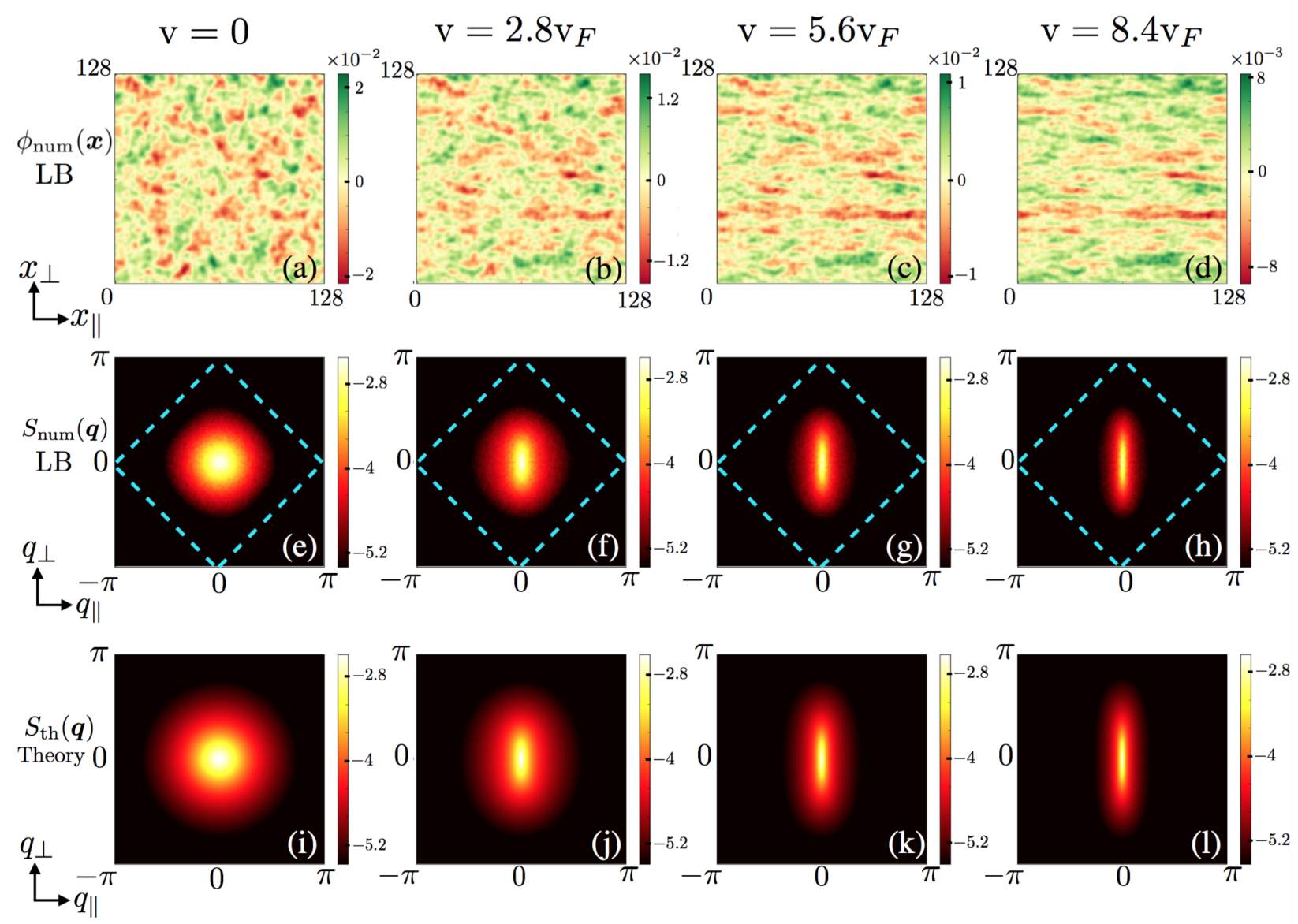

FIG. 8. (Color online) Comparisons between the predicted two-dimensional structure factor $S_{\text {th }}\left(q_{\|}, q_{\perp}\right)$ in the third row ((i)-(l)) and the simulated structure factor $S_{\text {num }}\left(q_{\|}, q_{\perp}\right)$ in the second row ((e)-(h)) from the Lattice Boltzmann (LB) simulations with $128^{2}$ sites. Typical simulated steady-state density fluctuations $\phi_{\text {num }}\left(x_{\|}, x_{\perp}\right)$ from the same disorder realization are shown in the first row ((a)-(e)) . The coordinate $\boldsymbol{x}=\left(x_{\|}, x_{\perp}\right)$ wavevector $\boldsymbol{q}=\left(q_{\|}, q_{\perp}\right)$ are displayed in the original (dimensional) scale with the lattice unit $l_{0}$ set to 1 . Simulation results are obtained from averaging the structure factors of density fluctuations over 200 disorder realizations. Color codes for the structure factors ((e)-(l)) are displayed in base-10 logarithmic scale. To highlight the agreements between the predicted long wavelength modes and the simulated ones, the values below $10^{-3}$-times the maximum are all represented in black; the predicted long-wavelength modes of $S_{\mathrm{th}}(q)$ are in excellent agreement with those of the simulated $S_{\text {num }}(q)$. The next-nearest neighbor effect of the D2Q9 scheme represented by the dashed blue diamonds in $S_{\text {num }}\left(q_{\|}, q_{\perp}\right)$ is similar to that displayed in Fig. 5, and is many order of magnitude smaller than the long-wavelength modes. At strong advection (right columns), the longitudinal long-wavelength modes are even more pronounced while the transverse long-wavelength modes are not significantly affected; this longitudinal compression in the structure factors indicates the formation of striated population structure exemplified in (c) and (d). The parameters in this figure are $D=5 \times 10^{-4}, a_{0}=9 \times 10^{-5}, \Delta=0.1 a_{0}$, and $b=10^{-9}$, which give $\xi_{D}=\sqrt{D / a_{0}} \approx 2.36, \mathrm{v}_{F}=2 \sqrt{D a_{0}} \approx 4.2 \times 10^{-4}$, and $\bar{K}=a_{0} / b=9 \times 10^{4}$.

lation structures might arise in natural marine microbial populations in spatially disordered 
growth environments and transported along closed recirculating flows, such as oceanic flows characterized by coherent Lagrangian vortices discussed in Refs. [5.5, 56].

In two-dimensional open flows, where populations do not actually recirculate, we numerically observe (not shown in this paper) the pinning-depinning phenomena of the population frontier, the interface between occupied and unoccupied regions, analogous to those observed in autocatalytic chemical reaction front propagating in a porous media with a background fluid flow [57-5.9]. In particular, the population frontier is pinned by growth rate disorder despite the presence of advection up to the critical advection speed, above which populations are completely washed away from the domain of interest. It would be interesting to explore, using the Lattice Boltzmann method, whether this pinning-depinning transition is a dynamic critical phenomena and, if so, whether it belongs to the universality class studied in Refs. [57-5.9].

\section{ACKNOWLEDGMENTS}

We thank Séverine Atis for useful discussions on pinning-depinning phenomena. This work was supported in part by the National Science Foundation (NSF) through Grant No. DMR-1306367 and by the Harvard Materials Research Science and Engineering Laboratory, through Grant No. DMR-1420570. Portions of this research were conducted during a stay at the Center for Models of Life at the Niels Bohr Institute, the University of Copenhagen. Computations were performed on the Odyssey cluster supported by the FAS Division of Science Research Computing Group at Harvard University. S.S. was supported by the Institute for Applied Computational Science at John A. Paulson School of Engineering and Applied Sciences at Harvard University, and by the Integrated Mesoscale Architectures for Sustainable Catalysis 389 (IMASC) Energy Frontier Research Center (EFRC) of the Department of Energy, Basic Energy Sciences, Award number 390 DE-SC0012573.

\section{Appendix A: Green's Functions, correlation functions, and structure factors}

This appendix contains calculation details of analytical results obtained in this paper. First, we solve Eq. ([7) by a direct Fourier transformation, as a check on Eq. (18). Upon setting $\left(\Delta / a_{0}\right)$ to unity for convenience, the Green's function of Eq. (17) in Fourier-space 
reads

$$
G(\tilde{\boldsymbol{q}})=\frac{1}{\tilde{q}^{2}-2 i \tilde{\mathrm{v}} \tilde{q}_{\|}+1} .
$$

Inverse fourier transformation gives the real-space Green's function in $d$-dimensions:

$$
G(\tilde{\boldsymbol{x}})=\int \frac{d^{d} \tilde{q}}{(2 \pi)^{d}} \frac{e^{i \tilde{\boldsymbol{q}} \cdot \tilde{\boldsymbol{x}}}}{\tilde{q}^{2}-2 i \tilde{\mathrm{v}} \tilde{q}_{\|}+1}
$$

To evaluate ( $\mathrm{A2})$, we use a trick, often employed to calculate correlation functions in field theory [60] , to convert the denominator into an exponential integral:

$$
\frac{1}{X^{n}}=\int_{0}^{\infty} d s \frac{s^{n-1} e^{-s X}}{(n-1) !} .
$$

With $n=1$, (A2) becomes

$$
\begin{aligned}
& G(\tilde{\boldsymbol{x}})=\int_{0}^{\infty} d s \int \frac{d^{d} \tilde{q}}{(2 \pi)^{d}} e^{i \tilde{\boldsymbol{q}} \cdot \tilde{\boldsymbol{x}}} e^{-s\left(\tilde{q}^{2}-2 i \tilde{\mathrm{v}} \tilde{q}_{\|}+1\right)}, \\
& =\int_{0}^{\infty} d s e^{-s} \underbrace{\left(\int \frac{d^{d-1}}{(2 \pi)_{\perp}} e^{i-1} e^{i \tilde{\boldsymbol{q}}_{\perp} \cdot \tilde{\boldsymbol{x}}_{\perp}-s \tilde{q}_{\perp}^{2}}\right)}_{\frac{1}{(2 \pi)^{d-1}} \sqrt{\frac{\pi}{s}}^{d-1} \exp \left(-\tilde{x}_{\perp}^{2} / 4 s\right)} \\
& \times \underbrace{\left(\int \frac{d \tilde{q}_{\|}}{(2 \pi)} e^{i \tilde{q}_{\|}\left(\tilde{x}_{\|}-2 s \tilde{\mathrm{v}}\right)-s \tilde{q}_{\|}^{2}}\right)}_{\frac{1}{(2 \pi)} \sqrt{\frac{\pi}{s}} \exp \left[-\left(\tilde{x}_{\|}-2 s \tilde{\mathrm{v}}\right)^{2} / 4 s\right]}, \\
& =\frac{\exp \left(\tilde{\mathrm{v}} \tilde{x}_{\|}\right)}{(2 \pi)^{d / 2}} \int_{0}^{\infty} d s s^{-d / 2} e^{-s\left(1+\tilde{\mathrm{v}}^{2}\right)-\tilde{x}^{2} / 4 s} .
\end{aligned}
$$

Upon recalling the integral representation of the modified Bessel function of a second kind [61]

$$
K_{\nu}(z)=\frac{1}{2}\left(\frac{1}{2} z\right)^{\nu} \int_{0}^{\infty} d t t^{-\nu-1} e^{-t-z^{2} / 4 t}
$$

and using the symmetry $K_{-\nu}(z)=K_{\nu}(z)$, (A4) becomes

$$
G(\tilde{\boldsymbol{x}})=\frac{\exp \left(\tilde{\mathrm{v}} \tilde{x}_{\|}\right)}{(\pi)^{d / 2}}\left[\left(\frac{|\tilde{\boldsymbol{x}}|}{\sqrt{1+\tilde{\mathrm{v}}^{2}}}\right)^{1-d / 2} K_{1-d / 2}\left(\sqrt{1+\tilde{\mathrm{v}}^{2}}|\tilde{\boldsymbol{x}}|\right)\right]
$$

which is identical to Eq. (18) with the gauge transformed Green's function $G_{\tilde{\mathrm{v}}}(\tilde{\boldsymbol{x}})$ of Eq. (20) by the square bracket (after restoring the factor $\Delta / a_{0}$ ). 
We now outline the details of calculations to achieve analytical results in Sec. WD that follow from the general prescription of the two-point correlation function of Eq. ([30), where again we set $\Delta / a_{0}=1$ for short. For $d=1$, the correlation function reads

$$
\langle\phi(\tilde{x}) \phi(0)\rangle=\frac{1}{6 \pi} \int_{-\infty}^{\infty} d \tilde{q} e^{i \tilde{q} \tilde{x}} \prod_{k=1}^{4} \frac{1}{\left(\tilde{q}-\tilde{q}_{k}\right)}
$$

where the four poles, that arise from the factorization of $\left[\left(\tilde{q}^{2}+1\right)^{2}+4 \tilde{\mathrm{v}}^{2} \tilde{q}^{2}\right]$, are located at

$$
\begin{aligned}
& \tilde{q}_{1}=-\tilde{q}_{2}=i\left(\sqrt{1+\tilde{\mathrm{v}}^{2}}+\tilde{\mathrm{v}}\right), \\
& \tilde{q}_{3}=-\tilde{q}_{4}=i\left(\sqrt{1+\tilde{\mathrm{v}}^{2}}-\tilde{\mathrm{v}}\right) .
\end{aligned}
$$

Closing the contour in the upper (lower) half plane for $\tilde{x}>0(\tilde{x}<0)$, then using the residue theorem yields the exact result of the one-dimensional correlation function given by Eq. (31)

For $d=2$, we first calculate the transverse two-point correlation function:

$$
\begin{aligned}
\left\langle\phi\left(0, \tilde{x}_{\perp}\right) \phi(\mathbf{0})\right\rangle & \sim \int \frac{d \tilde{q}_{\perp}}{2 \pi} e^{i \tilde{q}_{\perp} \tilde{x}_{\perp}} \int \frac{d \tilde{q}_{\|}}{2 \pi} \frac{1}{\left[\left(\tilde{q}^{2}+1\right)^{2}+4 \tilde{\mathrm{v}}^{2} \tilde{q}_{\|}^{2}\right]} \\
& =\frac{1}{4 \pi} \int d \tilde{q}_{\perp} \frac{e^{i \tilde{q}_{\perp} \tilde{x}_{\perp}}}{\left(\tilde{q}_{\perp}^{2}+1\right)\left(\tilde{q}_{\perp}^{2}+1+\tilde{\mathrm{v}}^{2}\right)^{1 / 2}}
\end{aligned}
$$

In the limit $\tilde{\mathrm{v}} \rightarrow \infty$, we obtain

$$
\begin{aligned}
\lim _{\tilde{\mathrm{v}} \rightarrow \infty}\left\langle\phi\left(0, \tilde{x}_{\perp}\right) \phi(\mathbf{0})\right\rangle & \sim \frac{1}{4 \pi \tilde{\mathrm{v}}} \int d \tilde{q}_{\perp} \frac{e^{i \tilde{q}_{\perp} \tilde{x}_{\perp}}}{\left(\tilde{q}_{\perp}^{2}+1\right)}, \\
& =\frac{e^{-\left|\tilde{x}_{\perp}\right|}}{4 \tilde{\mathrm{v}}}
\end{aligned}
$$

Now consider the longitudinal two-point correlation function.

$$
\begin{aligned}
\left\langle\phi\left(\tilde{x}_{\|}, 0\right) \phi(\mathbf{0})\right\rangle & \sim \int \frac{d \tilde{q}_{\|}}{2 \pi} e^{i \tilde{q}_{\|} \tilde{x}_{\|}} \int \frac{d \tilde{q}_{\perp}}{2 \pi} \frac{1}{\left[\left(\tilde{q}^{2}+1\right)^{2}+4 \tilde{\mathrm{v}}^{2} \tilde{q}_{\|}^{2}\right]}, \\
& =\int \frac{d \tilde{q}_{\|}}{2 \pi} e^{i \tilde{q}_{\|} \tilde{x}_{\|}}\left\{\frac{1}{8 i \tilde{\mathrm{v}}\left|\tilde{q}_{\|}\right|}\left[\frac{1}{\sqrt{f_{+}\left(\tilde{q}_{\|}\right)}}-\frac{1}{\sqrt{f_{-}\left(\tilde{q}_{\|}\right)}}\right]\right\},
\end{aligned}
$$


where the term in the curly bracket is the result of contour integration with respect to $\tilde{q}_{\perp}$ with the contour in the upper (lower) half plane for $\tilde{q}_{\|}>0\left(\tilde{q}_{\|}<0\right)$, and $f_{ \pm}\left(\tilde{q}_{\|}\right) \equiv\left(\tilde{q}_{\|}^{2}+1\right) \pm 2 i \tilde{\mathrm{v}} \tilde{q}_{\|}$. Because the integrand is real, the resulting integral must be real although it manifestly contains an imaginary part. To rewrite the squared bracket explicitly as a real-function, consider the following change of variable: $r e^{i \theta_{ \pm}} \equiv f_{ \pm}\left(\tilde{q}_{\|}\right)$. Then, $r$ and $\theta_{ \pm}$are given by $r=f_{+}\left(\tilde{q}_{\|}\right) f_{-}\left(\tilde{q}_{\|}\right)=\left(\tilde{q}_{\|}^{2}+1\right)^{2}+4 \tilde{\mathrm{v}}^{2} \tilde{q}_{\|}^{2}$, and $\theta_{+}=-\theta_{-}$with $\tan \left(\theta_{+}\right)=2 \tilde{\mathrm{v}} \tilde{q}_{\|} /\left(\tilde{q}_{\|}^{2}+1\right)$. After some algebra and trigonometric identities, one finds the term in the curly bracket of (A]1) becomes

$$
\left[\frac{1}{4 i r\left|\sin \left(\theta_{+}\right)\right|} \frac{1}{\sqrt{r}}\left(e^{-i \theta_{+} / 2}-e^{-i \theta_{-} / 2}\right)\right]=\frac{1}{2 \sqrt{2}} \frac{r^{-3 / 4}}{\sqrt{1+\cos \left(\theta_{+}\right)}}
$$

Substituting the expression into ([A]1), we obtain

$$
\begin{aligned}
& \left\langle\phi\left(\tilde{x}_{\|}, 0\right) \phi(\mathbf{0})\right\rangle=\int \frac{d \tilde{q}_{\|}}{4 \sqrt{2} \pi} e^{i \tilde{q}_{\|} \tilde{x}_{\|}} \\
& \times \frac{1}{\left[\left(\tilde{q}_{\|}^{2}+1\right)^{2}+4 \tilde{\mathrm{v}}^{2} \tilde{q}_{\|}^{2}\right]^{\frac{3}{4}}} \frac{1}{\left\{1+\left(1+\tilde{q}_{\|}^{2}\right) /\left[\left(\tilde{q}_{\|}^{2}+1\right)^{2}+4 \tilde{\mathrm{v}}^{2} \tilde{q}_{\|}^{2}\right]^{\frac{1}{2}}\right\}^{\frac{1}{2}}} .
\end{aligned}
$$

In the limit $\tilde{\mathrm{v}} \rightarrow \infty$, one obtains the longitudinal correlation function

$$
\begin{aligned}
\lim _{\tilde{\mathrm{v}} \rightarrow \infty}\left\langle\phi\left(\tilde{x}_{\|}, 0\right) \phi(\mathbf{0})\right\rangle & \sim \frac{1}{4 \sqrt{2} \pi} \int d \tilde{q}_{\|} \frac{e^{i \tilde{q}_{\|} \tilde{x}_{\|}}}{\left(1+4 \tilde{\mathrm{v}}^{2} \tilde{q}_{\|}^{2}\right)^{\frac{3}{4}}} \\
& =\frac{-2^{5 / 4}}{\tilde{\mathrm{v}} \pi \Gamma(-1 / 4)}\left(\frac{\left|\tilde{x}_{\|}\right|}{2 \tilde{\mathrm{v}}}\right)^{1 / 4} K_{-1 / 4}\left(\frac{\left|\tilde{x}_{\|}\right|}{2 \tilde{\mathrm{v}}}\right)
\end{aligned}
$$

which gives (35).

[1] R. M. May and R. M. Anderson, Parasitology 100, S89 (1990).

[2] N. J. Gotelli, A Primer of Ecology. (Sinauer Associates, Inc, 2001).

[3] J. D. Murray, Mathematical Biology II: Spatial Models and Biomedical Applications (SpringerVerlag New York Incorporated, 2001).

[4] M. Rietkerk, S. C. Dekker, P. C. de Ruiter, and J. van de Koppel, Science 305, 1926 (2004).

[5] M. Rietkerk and J. Van de Koppel, Trends in Ecology and Evolution 23, 169 (2008). 
[6] E. O. Budrene and H. C. Berg, Nature 349, 630 (1991).

[7] M. P. Brenner, L. S. Levitov, and E. O. Budrene, Biophysical Journal 74, 1677 (1998).

[8] M. Cates, D. Marenduzzo, I. Pagonabarraga, and J. Tailleur, Proceedings of the National Academy of Sciences 107, 11715 (2010).

[9] Q.-X. Liu, E. J. Weerman, P. M. Herman, H. Olff, and J. van de Koppel, Proceedings of the Royal Society of London B: Biological Sciences 279, 2744 (2012).

[10] Q.-X. Liu, P. M. Herman, W. M. Mooij, J. Huisman, M. Scheffer, H. Olff, and J. van de Koppel, Nature communications 5 (2014).

[11] J. van de Koppel, M. Rietkerk, N. Dankers, and P. M. Herman, The American Naturalist 165, E66 (2005).

[12] J. Von Hardenberg, E. Meron, M. Shachak, and Y. Zarmi, Physical Review Letters 87, 198101 (2001).

[13] F. Borgogno, P. D'Odorico, F. Laio, and L. Ridolfi, Reviews of Geophysics 47 (2009).

[14] T. Tél, A. de Moura, C. Grebogi, and G. Károlyi, Physics Reports 413, 91 (2005).

[15] Z. Neufeld, Chaos: An Interdisciplinary Journal of Nonlinear Science 22, 037102 (2012).

[16] W. J. McKiver and Z. Neufeld, Physical Review E 79, 061902 (2009).

[17] S. Pigolotti, R. Benzi, M. H. Jensen, and D. R. Nelson, Physical Review Letters 108, 128102 (2012).

[18] R. Benzi, M. H. Jensen, D. R. Nelson, P. Perlekar, S. Pigolotti, and F. Toschi, The European Physical Journal Special Topics 204, 57 (2012).

[19] D. R. Nelson, Annual Review of Biophysics 41, 371 (2012).

[20] K. A. Dahmen, D. R. Nelson, and N. M. Shnerb, in Statistical Mechanics of Biocomplexity (Springer, 1999), pp. 124-151.

[21] K. A. Dahmen, D. R. Nelson, and N. M. Shnerb, Journal of Mathematical Biology 41, 1 (2000).

[22] M. M. Desai and D. R. Nelson, Theoretical Population Biology 67, 33 (2005).

[23] D. R. Nelson and N. M. Shnerb, Physical Review E 58, 1383 (1998).

[24] A. L. Lin, B. A. Mann, G. Torres-Oviedo, B. Lincoln, J. Käs, and H. L. Swinney, Biophysical Journal 87, 75 (2004).

[25] F. Lutscher, R. M. Nisbet, and E. Pachepsky, Theoretical Ecology 3, 271 (2010).

[26] E. Pachepsky, F. Lutscher, R. Nisbet, and M. Lewis, Theoretical Population Biology 67, 61 
(2005).

[27] J. M. Pringle, A. M. H. Blakeslee, J. E. Byers, and J. Roman, Proceedings of the National Academy of Sciences 108, 15288 (2011).

[28] F. Lutscher, E. McCauley, and M. A. Lewis, Theoretical Population Biology 71, 267 (2007).

[29] O. Kogan, K. O'Keeffe, D. Schneider, and C. R. Myers, arXiv preprint arXiv:1510.08987 (2015).

[30] Z. Neufeld and E. Hernández-García, Chemical and Biological Processes in Fluid Flows (World Scientific, 2009).

[31] R. A. Fisher, Annals of Eugenics 7, 355 (1937).

[32] A. Kolmogoroff, I. Petrovsky, and N. Piscounoff, Moscow Univ. Math. Bull. 1, 1 (1937).

[33] J. D. Murray, Mathematical Biology I: An Introduction (Springer-Verlag New York Incorporated, 2002).

[34] T. Neicu, A. Pradhan, D. Larochelle, and A. Kudrolli, Physical Review E 62, 1059 (2000).

[35] L. G. Molinari, Journal of Physics A: Mathematical and Theoretical 42, 265204 (2009).

[36] N. Hatano and D. R. Nelson, Physical Review B 58, 8384 (1998).

[37] N. Hatano and D. R. Nelson, Physical Review Letters 77, 570 (1996).

[38] N. M. Shnerb and D. R. Nelson, Physical Review Letters 80, 5172 (1998).

[39] D. A. Kessler and N. M. Shnerb, New Journal of Physics 11, 043017 (2009).

[40] R. Juhász, Journal of Statistical Mechanics: Theory and Experiment 2013, P10023 (2013).

[41] L. Geyrhofer and O. Hallatschek, Journal of Statistical Mechanics: Theory and Experiment 2013, P01007 (2013).

[42] A. R. Missel and K. A. Dahmen, Physical Review E 79, 021126 (2009).

[43] W. Zimmermann, M. Seesselberg, and F. Petruccione, Physical Review E 48, 2699 (1993).

[44] J. Buceta and K. Lindenberg, Physical Review E 68, 011103 (2003).

[45] M. Cross and H. Greenside, Pattern Formation and Dynamics in Nonequilibrium Systems (Cambridge University Press, 2009).

[46] E. Abrahams, 50 years of Anderson Localization, vol. 24 (World Scientific, 2010).

[47] C. A. Lugo and A. J. McKane, Physical Review E 78, 051911 (2008).

[48] A. J. McKane, T. Biancalani, and T. Rogers, Bulletin of Mathematical Biology 76, 895 (2014).

[49] S. Hassani, Mathematical Physics: a Modern Introduction to its Foundations (Springer Science, 2013). 
[50] M. Abramowitz and I. A. Stegun, Handbook of Mathematical Functions: with Formulas, Graphs, and Mathematical Tables (Courier Corporation, 1964).

[51] A. Cali, S. Succi, A. Cancelliere, R. Benzi, and M. Gramignani, Physical Review A 45, 5771 (1992).

[52] S. Succi, EPL (Europhysics Letters) 109, 50001 (2015).

[53] S. Succi, The Lattice-Boltzmann Equation (Oxford University Press, Oxford, 2001).

[54] S. Succi, International Journal of Modern Physics C 25 (2014).

[55] G. Haller and F. Beron-Vera, Journal of Fluid Mechanics 731, R4 (2013).

[56] G. Haller, Annual Review of Fluid Mechanics 47, 137 (2015).

[57] S. Atis, S. Saha, H. Auradou, D. Salin, and L. Talon, Physical Review Letters 110, 148301 (2013).

[58] S. Saha, S. Atis, D. Salin, and L. Talon, EPL (Europhysics Letters) 101, 38003 (2013).

[59] T. Gueudré, A. K. Dubey, L. Talon, and A. Rosso, Physical Review E 89, 041004 (2014).

[60] E. Zeidler, Quantum Field Theory II (2008).

[61] F. W. Olver, D. W. Lozier, R. F. Boisvert, and C. W. Clark, NIST Handbook of Mathematical Functions (Washington, DC, 2010). 